\title{
Colloquium: Ice rule and emergent frustration in particle ice and beyond
}

\author{
Antonio Ortiz-Ambriz \\ Departament de Física de la Matèria Condensada, \\ Universitat de Barcelona, 08028, Barcelona, Spain \\ and Institut de Nanociència i Nanotecnologia (IN $\left.{ }^{2} U B\right)$, \\ Universitat de Barcelona, 08028, Barcelona, Spain
}

\author{
Cristiano Nisoliø, Charles Reichhardt,and Cynthia J. O. Reichhardt \\ Theoretical Division and Center for Nonlinear Studies, Los Alamos National Laboratory, \\ Los Alamos, New Mexico 87545, USA
}

\author{
Pietro Tierno ${ }^{*}$ \\ Departament de Física de la Matèria Condensada, Universitat de Barcelona, \\ 08028, Barcelona, Spain, Institut de Nanociència i Nanotecnologia (IN²UB), \\ Universitat de Barcelona, 08028, Barcelona, Spain, \\ and Universitat de Barcelona Institute of Complex Systems (UBICS), \\ Universitat de Barcelona, 08028, Barcelona, Spain
}

\section{(published 30 December 2019)}

\begin{abstract}
Geometric frustration and the ice rule are two concepts that are intimately connected and widespread across condensed matter. The first refers to the inability of a system to satisfy competing interactions in the presence of spatial constraints. The second, in its more general sense, represents a prescription for the minimization of the topological charges in a constrained system. Both can lead to manifolds of high susceptibility and nontrivial, constrained disorder where exotic behaviors can appear and even be designed deliberately. This Colloquium describes the emergence of geometric frustration and the ice rule in soft condensed matter. It excludes the extensive developments of mathematical physics within the field of geometric frustration, but rather focuses on systems of confined microscopic or mesoscopic particles that emerge as a novel paradigm exhibiting spin degrees of freedom. In such systems, geometric frustration can be engineered artificially by controlling the spatial topology and geometry of the lattice, the position of the individual particle units, or their relative filling fraction. These capabilities enable the creation of novel and exotic phases of matter and also potentially lead toward technological applications related to memory and logic devices that are based on the motion of topological defects. The rapid progress in theory and experiments is reviewed and the intimate physical connections with other frustrated systems at different length scales are discussed.
\end{abstract}

DOI: 10.1103/RevModPhys.91.041003

\section{CONTENTS}

I. Introduction

II. The Ice Rule

A. From water ice to spin ice

B. Artificial spin ice systems

C. Ice rule and topology: Conceptual themes

III. The Ice Rule in Soft (Particle) Systems
A. Colloids as a model system
B. Artificial colloidal ice: Simulations
C. Experimental realization
D. Defect dynamics, grain boundaries, and logic gate
E. Effect of disorder, doping, and system memory
F. Kagome ice and its inner phase
G. Other geometries

IV. The Fragile Ice Manifold

A. Nature of the ice rule in colloidal ice
B. Decimated systems

C. Discrete versus continuum models 15

D. Future directions in particle ice 16

V. Other Particle-based Frustrated Systems 17 VI. Outlook 19

Acknowledgments $\quad 20$

References 20

\section{INTRODUCTION}

Frustration in life emerges with the impossibility of simultaneously satisfying a set of requirements. Frustration in physics is not very different. A classical example is that of three spins on the vertex of a triangle that want to be antiferromagnetically aligned (Wannier, 1950). This requirement cannot be realized all around the triangle, so at least two spins will display ferromagnetic order and will generate one frustrated bond. More generally, a geometrically frustrated

ptierno@ub.edu 
system is one that is subjected to local requirements that cannot be satisfied collectively along certain loops in the system. The notion is therefore intrinsically topological, i.e., invariant under transformations that do not rip those frustrated loops, and indeed it lends itself to abstract, elegant treatments in term of Wilson loops and gauge symmetry, at least in the case of spin systems (Fradkin, Huberman, and Shenker, 1978). In real systems, however, the local requirement is usually the minimization of an energy as a pairwise interaction that is in general geometry dependent.

Frustration is essential for the understanding of a variety of real materials, such as spin glasses (Mydosh, 2014), water ice (Bernal and Fowler, 1933; Pauling, 1935), spin ices (Harris et al., 1997; Ramirez et al., 1999; Bramwell and Gingras, 2001; Skjaervø et al., 2019), and even systems at different length scales such as granular materials (Richard et al., 2005), liquid crystals (Kamien and Selinger, 2001; Lopez-Leon et al., 2011), filament bundles (Hall et al., 2016), coupled lasers (Nixon et al., 2013), and many others (Grason, 2016). It is often, but by no means always, associated with slow relaxation, degeneracy, and zero temperature entropy. Indeed, frustration implies compromise and therefore produces various forms of so-called constrained disorder, which are manifolds whose disorder obeys some nontrivial rules, either local or global. The ice rule is an important example of a local rule that constrains disorder and that can have global, i.e., topological, implications.

Typically in an ensemble characterized by constrained disorder, violations of local rules appear as localized excitations of the low-energy states of the system. These local rule violations control the collective dynamics. An example, described later, is provided by the magnetic monopoles in spin ices (Ryzhkin, 2005; Castelnovo, Moessner, and Sondhi, 2008), which are violations of an ice manifold. Another example is the emergent topological charges in certain artificial spin ices (Lao et al., 2018). These concepts are both of practical interest, such as for the measurement of currents of magnetic monopoles (Giblin et al., 2011), and of theoretical interest. Indeed, we usually understand topological defects in terms of alterations of an underlying order, such as misplaced books on a library shelf or dislocations in a crystal, rather than in terms of disorder.

Manifolds of constrained disorder can be rich playgrounds for new physics, and frustration is a fundamental ingredient in the design of artificial systems that can generate novel exotic behaviors which are often not found in natural materials (Wang et al., 2006; Heyderman and Stamps, 2013; Nisoli, Moessner, and Schiffer, 2013). Artificial spin ice systems were first created by mimicking natural frustrated geometries and by realizing celebrated models of statistical mechanics (Lieb, 1967b; Baxter, 1982) in settings that allowed characterization at the constituent level, often in real time. However, since artificial materials can be realized in various geometries, a more recent effort (Morrison, Nelson, and Nisoli, 2013; Nisoli, Kapaklis, and Schiffer, 2017) has advanced the design of new systems generating a wide variety of new phenomena, including dimensionality reduction, emergent classical topological order, realizations of Pott's models, phase transitions, ice rule fragility, and quasicrystal spin ices (Gilbert et al., 2014, 2016; Ma et al., 2016; Perrin, Canals, and Rougemaille,
2016; Gliga et al., 2017; Lao et al., 2018; András Libál et al., 2018; Louis et al., 2018; Östman et al., 2018; Shi et al., 2018; Barrows et al., 2019; Sklenar et al., 2019). Furthermore, many of these ideas proved to be exportable across different platforms, from nanomagnets to trapped colloids, to liquid crystals, and to superconductors (Libál, Olson Reichhardt, and Reichhardt, 2009; Latimer et al., 2013; Ortiz-Ambriz and Tierno, 2016; Wang et al., 2018; Duzgun and Nisoli, 2019).

\section{THE ICE RULE}

We start by briefly recalling the history of the ice rule and its appearance in magnetic systems either natural or lithographically fabricated. For a more extensive treatment we refer the interested reader to available reviews and commentaries on the subject (Ramirez, 1994; Bramwell and Gingras, 2001; Gardner, Gingras, and Greedan, 2010; Heyderman and Stamps, 2013; Nisoli, Moessner, and Schiffer, 2013; Gilbert, Nisoli, and Schiffer, 2016; Nisoli, 2018a).

\section{A. From water ice to spin ice}

Many fundamental properties of water are still not completely understood (Chaplin, 2006). One of water's early mysteries pertained to its residual entropy and was solved by Linus Pauling in the 1930s. Through a series of carefully conducted calorimetric experiments, Giauque and Ashley (1933) and Stout and Giauque (1936) found that the entropy of ice at low temperature was not zero. Pauling (1935) explained it via the ice rule of Bernal and Fowler (1933). In water ice each oxygen atom is bound to four others via hydrogen atoms. Two of these hydrogen atoms are close to the oxygen atom in covalent bonds, and two are farther away forming covalent bonds with neighboring oxygen atoms; see Fig. 1(a), left. The number of ways in which the hydrogen atoms can be arranged, Pauling showed, grows exponentially with the number of oxygen atoms, leading to a nonzero entropy per molecule. Icelike systems received renewed interest in the 1990s with the discovery of a class of magnetic substances which were named "spin ices" because their magnetic texture at low temperature presents a degenerate low-energy state with a residual entropy, consistent with the ice rule model. These rare earth titanates, such as $\mathrm{Ho}_{2} \mathrm{Ti}_{2} \mathrm{O}_{7}$ and $\mathrm{Dy}_{2} \mathrm{Ti}_{2} \mathrm{O}_{7}$, presented frustrated interactions between the magnetic moments of their constituent at low temperature. Their magnetic cations $\mathrm{Ho}^{3+}$ and $\mathrm{Dy}^{3+}$ were known to carry a very large magnetic moment, about 10 times the Bohr magneton. At low temperatures such moments could be regarded as binary, classical Ising spins constrained to point along the directions of the lattice bonds forming the pyrochlore lattice, as shown in Fig. 1(a), right. It was then noted (Harris et al., 1997) and confirmed experimentally (Ramirez et al., 1999) that the resulting ferromagnetic interaction favors the ice rule, in which two spins point in within each vertex and two out, in analogy with the allocation of protons in the lattice of water ice. 

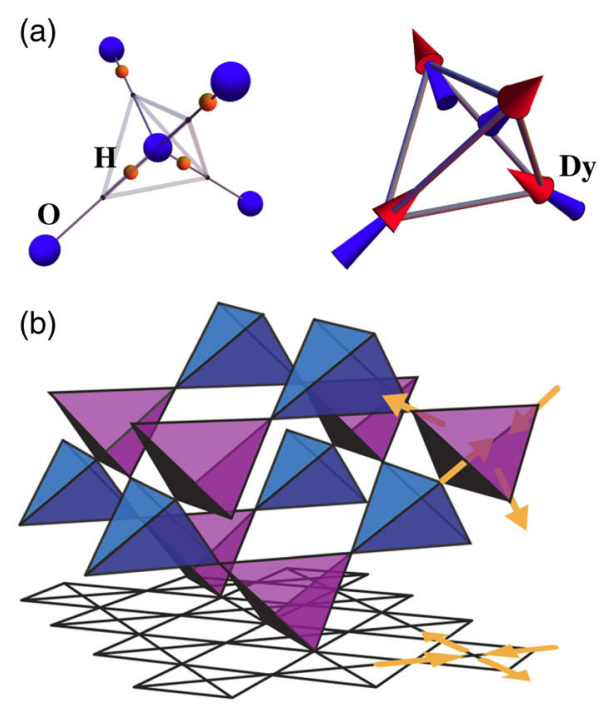

(c)

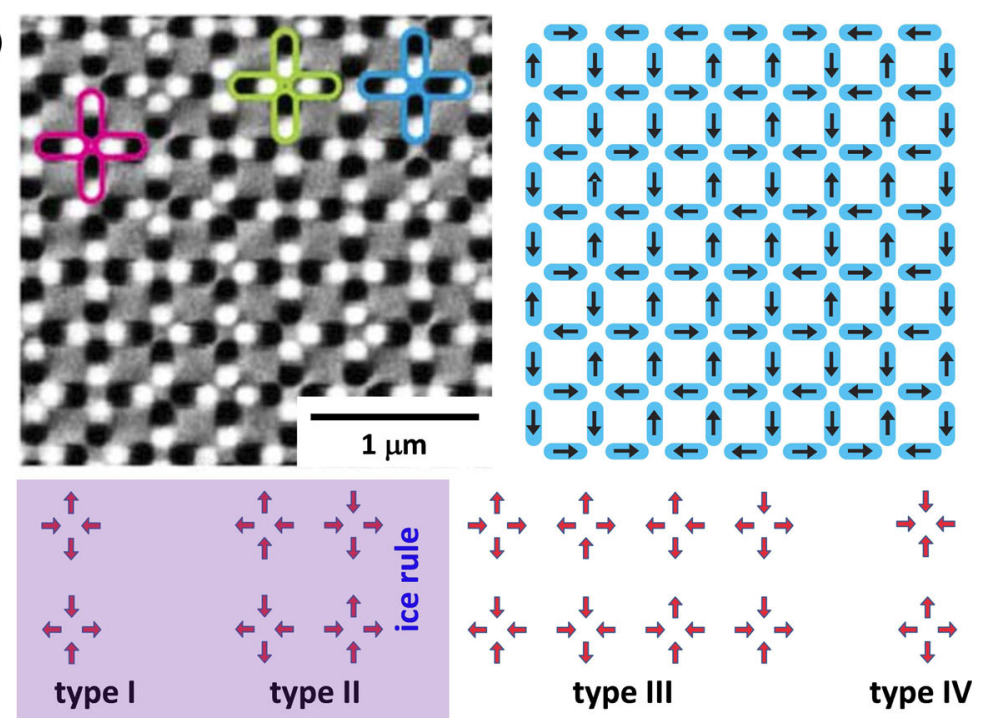

FIG. 1. (a) Ice rule for the water ice $\mathrm{I}_{h}$ (left) and magnetic spin ice material (right). The first is characterized by oxygen atoms sharing protons and located at the vertices of a diamond lattice. The spin ice has Ising-like moments pointing along the same lattice. From Castelnovo, Moessner, and Sondhi, 2012. (b) A projection of the three-dimensional pyrochlore lattice onto a two-dimensional plane gives rise to a square lattice. From Glaetzle et al., 2014. (c) Left: Magnetic force microscope image of an artificial spin ice composed of a square lattice of permalloy islands with lattice spacing of $400 \mathrm{~nm}$. Right: Corresponding spin orientations. Bottom: Vertex configurations with the ice rule highlighted in the violet (gray) box. From Wang et al., 2006.

\section{B. Artificial spin ice systems}

Between the late 1990s and the early 2000s, a mature effort in the exploration of the magnetic state of nanodots and nanoislands (Bader, 2006) focused on how to obtain exotic magnetic textures and transitions. Meanwhile, a different approach to exotic behavior involved relatively simple elongated, single-domain, magnetic nanoislands, whose magnetization could be described by an Ising pseudospin. In this case, complexity is introduced via the mutual interaction of these nanoislands in order to generate possibly interesting collective states of exotic emergent behaviors (Libál, Reichhardt, and Olson Reichhardt, 2006; Tanaka et al., 2006; Wang et al., 2006; Heyderman and Stamps, 2013; Nisoli, Moessner, and Schiffer, 2013). This approach provided a twofold advantage. First, characterization of the individual degrees of freedom in real space is possible via methods such as magnetic force microscopy (MFM), photoelectron emission microscopy, transmission electron microscopy, surface magneto-optic Kerr effect, and Lorentz microscopy. Second, the collective behavior of these systems is open to design.

The resulting so-called artificial spin ices (ASI) could mimic the behavior of spin ice rare earth pyrochlores, hence the name, but at desirable temperature and field ranges. In a certain geometry, ASI represents a plane projection of a pyrochlore material, as shown in Figs. 1(b) and 1(c). Soon a growing number of groups began using ASI to investigate topological defects, the dynamics of magnetic charges, and spin fragmentation (Ladak et al., 2010; Ladak, Read, Branford, and Cohen, 2011; Ladak, Read, Tyliszczak et al., 2011; Mengotti et al., 2011; Phatak et al., 2011; Rougemaille et al., 2011; Pollard, Volkov, and Zhu, 2012; Zeissler et al., 2013; Canals et al., 2016; Rougemaille and Canals, 2019), as well as information encoding (Lammert et al., 2010; Wang et al., 2016), equilibrium and nonequilibrium thermodynamics (Nisoli et al., 2007, 2010; Ke et al., 2008; Budrikis, Politi, and Stamps, 2011; Morgan et al., 2011; Lammert, Crespi, and Nisoli, 2012; Nisoli, 2012; Budrikis et al., 2013; Levis et al., 2013; Chioar, Canals et al., 2014; Cugliandolo, 2017), avalanches (Mellado et al., 2010; Hügli et al., 2012), direct realizations of the Ising system (Zhang et al., 2012; Chioar, Rougemaille et al., 2014; Arnalds et al., 2016; Chioar, Rougemaille, and Canals, 2016; Nisoli, 2016), magnetoresistance and the Hall effect (Branford et al., 2012; Le et al., 2017), critical slowing down (Anghinolfi et al., 2015), dislocations (Drisko, Marsh, and Cumings, 2017), magnetization dynamics (Lendinez and Jungfleisch, 2020), spin wave excitations (Gliga et al., 2013), ratchet effects (Gliga et al., 2017), dimensionality reduction (Gilbert et al., 2016), classical topological states (Gilbert et al., 2014; Perrin, Canals, and Rougemaille, 2016; Lao et al., 2018), quasicrystals (Shi et al., 2018; Barrows et al., 2019), and memory effects (Libál, Reichhardt, and Olson Reichhardt, 2012; Gilbert et al., 2015).

\section{Ice rule and topology: Conceptual themes}

The ice rule appears an innocuous enough concept, yet it can be understood in the most general terms and has profound implications. Consider a lattice or even a directed graph with binary variables such as Ising spins on each edge, impinging in vertices of various coordination $z$. The pyrochlore or square geometries introduced before can serve as examples. Then we can define the topological charge of a vertex of coordination $z$ with $n$ spins pointing toward it as

$$
q=2 n-z
$$

which corresponds to the difference between the number of spins pointing in and out. This notion is properly topological 
as it depends only on the topology of the graph. Furthermore, given a spin configuration, any single spin flip will alter the charge in two nearby vertices. Only flipping proper loops of spins will preserve the charge distribution.

In this language, the ice rule can be considered a prescription for a local minimization of $|q|$ at each vertex. In practical systems this is typically (but not necessarily) enforced by the nearest neighboring spin-spin interactions. Any configuration of spins that locally minimizes $|q|$ is said to obey the ice rule, and the subset of the phase space corresponding to such configurations is called an ice manifold. For a lattice of uniform, even coordination the ice manifold is then characterized by zero charge $q=0$ on each vertex. The first violation of the ice rule then corresponds to charges $q= \pm 2$, called monopoles. Very often, but not always, the ice manifold represents the lowest energy of the system, and then the ground state is degenerate and disordered and has a residual entropy. Importantly it cannot be "explored from within." Any single spin flip on an ice rule configuration creates a pair of monopoles of opposite charge \pm 2 (Ryzhkin, 2005; Castelnovo, Moessner, and Sondhi, 2008) violating the ice rule. Only the coherent flipping of a loop of spins, properly chosen so that they are all arranged head to tail, represents an "update" that does not violate the ice rule.

The simplest, square version of such a system has motivated theoretical research in applied mathematics for half a century. In the 1960s, Lieb, Wu, Baxter, Rys, and others began working on simplified, two-dimensional models of mathematical physics, known as vertex models, that captured and also generalized the properties of ice. In these models, different energies are assigned to different vertex configurations on a square lattice, and in many cases the models can be solved exactly, typically via transfer matrix methods (Rys, 1963; Lieb, 1967a, 1967b; Wu, 1969; Baxter, 1982). The sixvertex model, in particular (Lieb, 1967b), admits only ice rule obeying vertices on a square lattice and was meant to represent a solvable, two-dimensional equivalent of water ice. As it forbids monopoles, it is completely embedded into the ice manifold and has strong topological properties. For instance, if the degeneracy of the ice manifold is lifted by an energetics that selects the antiferromagnetic state, as in the Rys F model (Rys, 1963), then the corresponding ordering transition is infinitely continuous (Lieb, 1967a), and with an order parameter, also infinitely continuous (Baxter, 1982).

Beside clarifying the topological nature of the ice rule, these models were also often equivalent to other important statistical mechanics systems, such as dimer cover models, and thus initiated an independent theoretical line of research in mathematical physics that has further evolved in terms of loop representations to describe topological effects at (and of) the boundaries. We will not report here on this half a century long, interesting development because it goes beyond the scope of this Colloquium. Indeed the interesting phenomenology in those models arises from a topological structure, given by the ice rule, that in simulations and experiments is always violated. While it is true that such topological structure is present in the ground state, this does not imply that it extrapolates fully to the low-energy physics, which is in fact generally a dynamics of topological defects. In a sense, realistic spin ice systems are neither topologically constrained nor unconstrained. They should perhaps be called "topology breaking" systems because, for any nonzero temperature, violations of the topological constraints (the ice rule) in the form of monopoles substantially change the physics. For a dramatic example, while the antiferromagnetic ground state of artificial square ice (Wang et al., 2006; Nisoli et al., 2010; Morgan et al., 2011; Porro et al., 2013; Zhang et al., 2013) would seem to be well described by the Rys F model, the ordering transition of the latter is in the Kosterlitz-Thouless class (Lieb, 1967a; Kosterlitz and Thouless, 1973), whereas in "real" square ice the transition is simply second order in the Ising class (Wu, 1969; Levis et al., 2013; Sendetskyi et al., 2019) precisely because monopoles break the topological constraint.

Thus in real spin ice systems it becomes less interesting to eviscerate all the possible topological representations of their idealized ice manifold. It is instead more interesting to see how the topological properties of an ultimately unreachable ground state affect the low-energy physics as the system breaks those topological constraints. This issue has been addressed via the concepts of spin fractionalization into magnetic charges (Ryzhkin, 2005; Castelnovo, Moessner, and Sondhi, 2008) and spin fragmentation (Petit et al., 2016) into a Coulomb (Henley, 2010) and a non-Coulomb magnetization. For instance, in pyrochlore ice the ice manifold can be considered as a classical topological phase of constrained disorder (Henley, 2010, 2011; Castelnovo, Moessner, and Sondhi, 2012). Such a phase is labeled not by an order parameter, as an ordered phase would, but rather by a fluctuating, solenoidal gauge field $\vec{M}(x)$, which can be thought of as a coarse graining of the spin magnetization. Then spin fractionalization implies that the low-energy manifold can be described in terms of local violations of its solenoidal nature, such that

$$
\vec{\nabla} \cdot \vec{M}(x)=4 \pi \sum_{i} q_{x_{i}} \delta\left(x-x_{i}\right)
$$

Thus, the ice manifold corresponds to $q=0$ everywhere, and it is easy to show (Henley, 2010) that it implies algebraic (specifically, dipolar) correlations in the reciprocal space, and thus the observed pinch points in the structure factor of neutron scattering. While this ice phase is critical, this is a purely mathematical abstraction since no transition to the ice manifold exists, and at nonzero temperature monopoles will always be present, however sparsely, and provide a correlation length.

The low energy of the system remains, however, reminiscent of the topological nature of its ground state. Through spin fragmentation (Petit et al., 2016) it is possible to show that the low-energy ensemble can be decomposed into an ice rule ensemble plus a crystal-charge ensemble. In terms of the coarse grained magnetization this merely corresponds to a Helmholtz decomposition of the magnetization vector in the sum of a divergence free and a divergence full part. Because the two components are uncoupled at the lowest order in the effective free energy, dipolar correlations and pinch points survive at nonzero temperature. Artificial realization of topological phases was achieved in the Shakti geometry (Lao et al., 
2018) as well as in magnetic square ice (Möller and Moessner, 2006; Perrin, Canals, and Rougemaille, 2016) and rectangular (Ribeiro et al., 2017; Loreto et al., 2019) ice. No equivalent phase has yet been realized with colloids, although those magnetic realizations provide directions.

For vertices of odd coordination, the cancellation of the charge in Eq. (1) is impossible and $|q|$ is minimized when $q= \pm 1$. Thus in a simple system of uniform, odd coordination, such as the kagome ice (Qi, Brintlinger, and Cumings, 2008), the ice manifold corresponds to ensembles of disordered spins obeying a 2-in/1-out or 2-out/1-in ice rule at each vertex. Because there is no charge cancellation, such systems can be regarded as disordered, neutral plasmas of opposite topological charges which, depending on realization, can interact, leading to further phases within the ice manifold (Möller and Moessner, 2009; Chern, Mellado, and Tchernyshyov, 2011; Chern, Morrison, and Nisoli, 2013; Zhang et al., 2013; Drisko, Daunheimer, and Cumings, 2015; A. Libál et al., 2018), as we show next in more detail.

Finally, systems of mixed coordination, say for instance $z=3,4$ host both versions of the ice rule, 2-in/2-out on $z=4$ coordinated vertices and 2-in/1-out or 2-out/1-in on $z=3$ vertices. Here is where the difference between magnetic and particle-based spin ice becomes more dramatic, with the ice rule breaking down in the latter, as we see in Sec. IV.

\section{THE ICE RULE IN SOFT (PARTICLE) SYSTEMS}

\section{A. Colloids as a model system}

Colloidal particles represent a versatile model system to explore collective phenomena in statistical physics and condensed matter, due to their accessible length scales and to the presence of simple and tunable interactions (Yethiraj and Blaaderen, 2003; Poon, 2004). Colloidal systems provided new insight into the glass transition (Weeks, 2017), yielding phenomena (Schall, Weitz, and Spaepen, 2007), and the motion of dislocations (Schall, 2004), and have been used to test numerous foundations of both equilibrium (Zahn, Lenke, and Maret, 1999; Thorneywork et al., 2017) and nonequilibrium statistical mechanics (Martínez et al., 2016, 2017). One of the best examples of the use of colloids for both condensed matter and statistical physics studies is in understanding ordering and commensuration effects on surfaces, where the substrate can be one dimensional (1D) (Bechinger, Brunner, and Leiderer, 2001), two dimensional (2D) (Brunner and Bechinger, 2002; Mangold, Leiderer, and Bechinger, 2003; Bohlein, Mikhael, and Bechinger, 2012), quasiperiodic (Mikhael et al., 2008), or random (Deutschländer et al., 2013). For colloids interacting with periodic 1D surfaces, various effects such as transitions among liquid, 2D hexagonal solid, and smectic states appear as a function of increasing substrate strength (Bechinger, Brunner, and Leiderer, 2001; Tierno et al., 2008; Tierno, 2012). The next level of complexity is to consider colloids interacting with two-dimensional periodic arrays, such as an egg carton (Reichhardt and Olson, 2002; Mangold, Leiderer, and Bechinger, 2003; Agra, van Wijland, and Trizac, 2004; Šarlah, Franosch, and Frey, 2005; Bohlein, Mikhael, and Bechinger, 2012), a muffin tin (Bechinger, Brunner, and Leiderer, 2001), or more complex potentials
(Gunnarsson et al., 2005; Yellen, Hovorka, and Friedman, 2005; Demirörs et al., 2013; Tierno, 2014; Tierno and Fischer, 2014; Loehr et al., 2016; de las Heras et al., 2016; Massana-Cid et al., 2019). Such systems can mimic the ordering of atoms on 2D surfaces (Coppersmith et al., 1982), vortices in type-II superconductors with nanostructured pinning (Baert et al., 1995; Harada et al., 1996; Martín et al., 1999), and vortices in Bose-Einstein condensates (Tung, Schweikhard, and Cornell, 2006) interacting with $2 \mathrm{D}$ optical trap arrays. In this case, commensuration effects arise when the number of colloids is an integer multiple of the number of potential minima, giving an integer filling factor $f$, where at $f=1$ each trap captures a single colloidal particle. Experiments (Bechinger, Brunner, and Leiderer, 2001; Bohlein, Mikhael, and Bechinger, 2012) and theoretical studies (Reichhardt and Olson, 2002; Agra, van Wijland, and Trizac, 2004; Šarlah, Franosch, and Frey, 2005) of colloids interacting with 2D substrates reveal a variety of novel orderings, including commensurate colloidal molecular crystals for $f=2,3, \ldots, N$. When the system is away from commensuration, such as just above $f=1.0$, the additional particles act like highly mobile kinks, and both simulations and experiments for colloids on 2D arrays have revealed the motion of these kinks under an applied drive (Bohlein, Mikhael, and Bechinger, 2012; Vanossi, Manini, and Tosatti, 2012; McDermott et al., 2013). Other studies of colloids have focused on the Aubry transitions that occur as a function of substrate strength (Brazda et al., 2018).

Since various substrates for colloidal particles can be readily created, including systems in which a single trap contains a double-well potential (Babič and Bechinger, 2005), a natural question to ask is whether it is possible to create a colloidal system containing effective spin degrees of freedom. For colloids on regular 2D arrays, it was shown that for integer $f=2$ and higher the colloids in each trap act like dimers with a spin degree of freedom that can be mapped to an Ising one, making it possible to use colloids in periodic substrate arrays to model various spin systems (Reichhardt and Olson, 2002; Agra, van Wijland, and Trizac, 2004; Šarlah, Franosch, and Frey, 2005).

\section{B. Artificial colloidal ice: Simulations}

Motivated by these ideas, and influenced by the work on magnetic ASI, Libál, Reichhardt, and Olson Reichhardt (2006) proposed that the $2 \mathrm{D}$ array of double-well traps for colloids could mimic a square ASI. In this case the unit cell consists of four double-well traps arranged as shown in Fig. 2(a), where $f=1$ so that each trap captures a single colloidal particle. The colloid-colloid interaction potential is of Yukawa or screened Coulomb form, $V(r) \propto q_{e} \exp (-\kappa r) / r$, where $q_{e}$ is the electrostatic charge and $1 / \kappa$ is the screening length.

The simulations for $N$ charged colloids on a 2D array of $N$ traps are performed using Brownian dynamics. The colloid dynamics are overdamped and the equation of motion for colloid $i$ is

$$
\eta \frac{d \mathbf{r}_{i}}{d t}=\mathbf{F}_{i}^{c c}+\mathbf{F}_{i}^{T}+\mathbf{F}_{i}^{\mathrm{ext}}+\mathbf{F}_{i}^{s}
$$

where in rescaled units the damping constant is set to $\eta=1.0$ and $a_{0}$ is used as the unit of distance in the simula- 


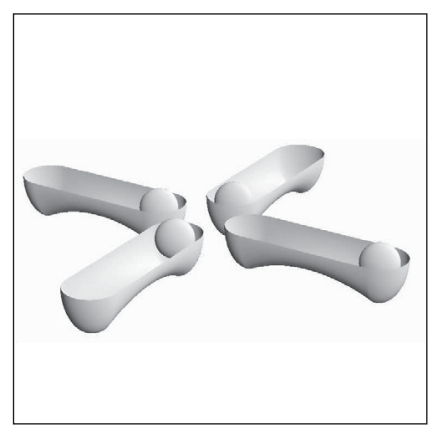

(a)

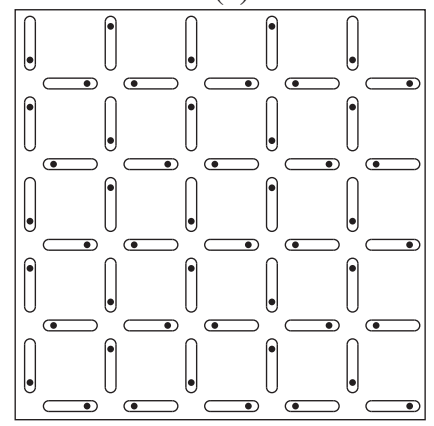

(c)

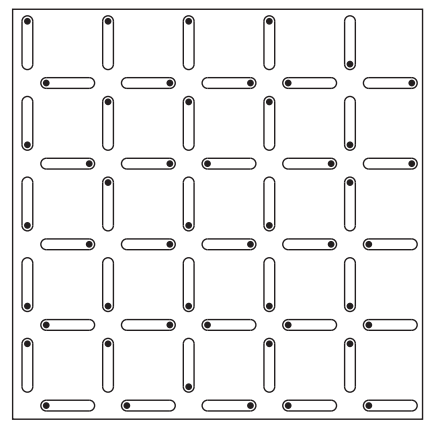

(b)

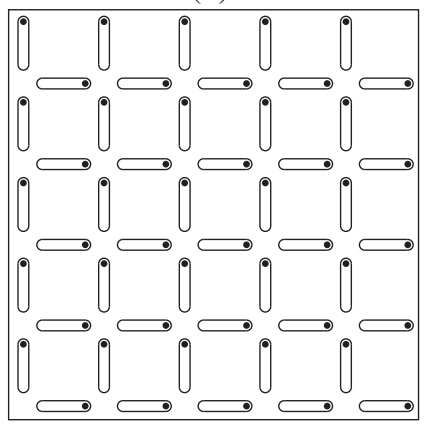

(d)
FIG. 2. (a) Schematic of the basic unit cell with four double wells each filled by one colloid. (b)-(d) Images of a small portion of the system for different electrostatic charges $q_{e}$. Dark circles: colloids; ellipses: traps. (b) A random vertex distribution at $q_{e}=0$. (c) A long-range ordered square ice ground state at $q_{e}=1.3$. (d) A biased system at $q_{e}=0.4$ where a drive of $F^{d c}=$ 0.02 has been applied at an angle of $45^{\circ}$ from the $\hat{\boldsymbol{x}}$ axis. From Libál, Reichhardt, and Olson Reichhardt, 2006.

tion. The colloid-colloid interaction force is given by $\mathbf{F}_{i}^{c c}=$ $-F_{0} \sum_{i \neq j}^{N} \nabla_{i} V\left(r_{i j}\right)$ with $V\left(r_{i j}\right)=\left(1 / r_{i j}\right) \exp \left(-\kappa r_{i j}\right) \hat{\mathbf{r}}_{i j}$. Here $r_{i j}=\left|\mathbf{r}_{i}-\mathbf{r}_{j}\right|, \hat{\mathbf{r}}_{i j}=\left(\mathbf{r}_{i}-\mathbf{r}_{j}\right) / r_{i j}, \quad \mathbf{r}_{i(j)}$ is the position of particle $i(j), F_{0}=q_{e}^{2} /\left(4 \pi \epsilon \epsilon_{0}\right), \epsilon$ is the solvent dielectric constant, and $\kappa=4 / a_{0}$ which is the typical screening length for charged colloidal systems. The substrate force $\mathbf{F}_{s}^{i}$ arises from the elongated traps shown in Fig. 2(a) and arranged on a square lattice; more details are described by Libál, Reichhardt, and Olson Reichhardt (2006). These simulations neglect hydrodynamic interactions between the colloids, which is a reasonable assumption for charged particles in the low volume fraction limit or where the dynamics are dominated by hopping events rather than large scale flows. The effects of thermal noise are captured in the simulation via the force term $\mathbf{F}^{T}$, which represents random Langevin kicks with the properties $\left\langle\mathbf{F}_{i}^{T}\right\rangle=0$ and $\left\langle\mathbf{F}_{i}^{T}(t) \mathbf{F}_{j}^{T}\left(t^{\prime}\right)\right\rangle=2 \eta k_{B} T \delta_{i j} \delta\left(t-t^{\prime}\right)$. Unless otherwise mentioned, $F^{T}=\left|\mathbf{F}^{T}\right|=0$.

For charged colloidal systems, a bias can be applied using an electric field in order to mimic the effect of an external magnetic field in ASI. In the simulation model, this biasing field is represented by the term $\mathbf{F}_{i}^{\text {ext }}$. If, for example, the field $\mathbf{F}_{i}^{\text {ext }}=F^{d c}(\hat{\boldsymbol{x}}+\hat{\boldsymbol{y}})$ is used to bias the colloids along a $45^{\circ}$ angle from the $\hat{x}$ axis, the ice state illustrated in Fig. 2(d) emerges.

In the absence of a substrate, charged colloids confined to two dimensions will form a triangular lattice (Kusner et al.,
1994). For the traps in Fig. 2(a), there are two equally favorable locations. The two resulting configurations can be mapped to a spin degree of freedom in which the spin is defined to point toward the end of the trap occupied by the colloid. The lowest energy state for the single unit cell shown in Fig. 2(a) has all the colloids sitting at the end of the trap that is farthest from the vertex, so that all the effective spins point away from the vertex. This minimizes the colloid-colloid interaction energy. The highest energy state has all of the colloids sitting close to the vertex, so that all of the effective spins point toward the vertex. This corresponds to a doubly charged monopole state.

As shown in Fig. 2(b), when many unit cells are assembled into a lattice, it is not possible for all vertices to adopt their lowest energy configuration simultaneously since the geometric arrangement competes with the symmetry of the pair interaction. If the electrostatic screening is very strong or the trapping sites are sufficiently far enough apart, the colloids do not interact with each other and the arrangement of the effective spins is random, as illustrated in Fig. 2(b), where the populations of the different possible vertex states match with what is expected for a purely thermal distribution. For strong colloid-colloid interactions, the system forms the lowest energy collective ground state, in which each vertex has two colloids close to it and two colloids far from it, equivalent to the ice rule of the square ASI (2-in/2-out); see Fig. 2(c). If a biasing field is applied at $45^{\circ}$ from the $\hat{\boldsymbol{x}}$ axis, the system forms a biased state with high-energy vertices which still obey the ice rule; see Fig. 2(d).

The system can be characterized according to the six possible vertex types, whose energies are listed in Table I. From the lowest to the highest energy, the vertex types are type I, with all four spins out to create a double monopole; type II, with one spin in and three spins out to create a monopole; type III, the spin ice rule obeying state; type IV, the biased ice rule state; type $\mathrm{V}$, with three spins in and one spin out to create a monopole; and type VI, with all four spins in to create a high-energy double monopole. An immediate difference from the magnetic version of square ASI is that, in the colloidal artificial ice, the monopole states II and V and the double monopole states I and VI have different energies. This becomes important in the mobility of the monopoles and is an indication of the fact that the particle-based spin ice system minimizes the global interaction energy rather than the local vertex energy.

Using numerical simulations of this geometry, Libál, Reichhardt, and Olson Reichhardt (2006) examined the evolution of the vertex populations when passing from the weak to the strong interacting limit. Figure 3(a) shows the fraction

TABLE I. Normalized electrostatic energy $E_{i} / E_{I I I}$ for each vertex type. An example configuration for each vertex is listed; 1 (0) indicates a colloid close to (far from) the vertex. From Libál, Reichhardt, and Olson Reichhardt, 2006.

\begin{tabular}{lclccc}
\hline \hline Type & Configuration & $E_{i} / E_{I I I}$ & Type & Configuration & $E_{i} / E_{I I I}$ \\
\hline I & 0000 & 0.001 & IV & 1001 & 7.02 \\
II & 0001 & 0.0214 & V & 1101 & 14.977 \\
III & 0101 & 1.0 & VI & 1111 & 29.913 \\
\hline \hline
\end{tabular}




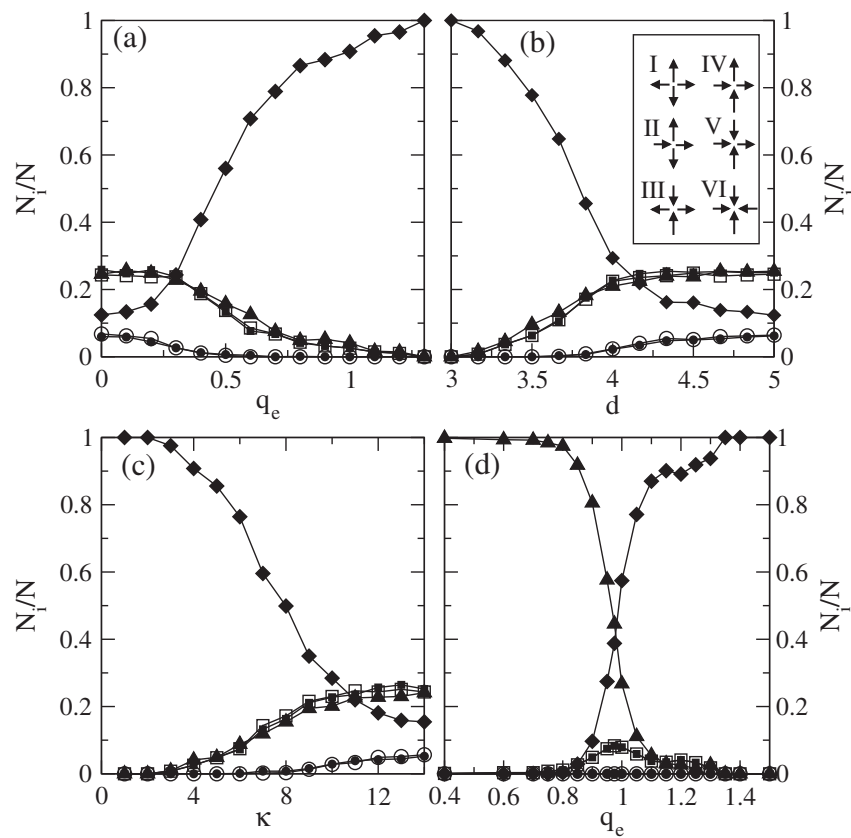

FIG. 3. Open circles: $N_{I} / N$; open squares: $N_{I I} / N$; black diamonds: $N_{I I I} / N$; black triangles: $N_{I V} / N$; black squares: $N_{V} / N$; black circles: $N_{V I} / N$. (a) $N_{i} / N$ vs $q_{e}$ at $d=3$ and $\kappa=4.0$. (b) $N_{i} / N$ vs $d$ at $q_{e}=1.3$ and $\kappa=4.0$. Inset: schematic spin representation of the six vertex types. (c) $N_{i} / N$ vs $\kappa$ at $d=3$ and $q_{e}=1.0$. (d) $N_{i} / N$ vs $q_{e}$ for a biased system at $d=3$, $\kappa=4.0$, and $F^{d c}=0.02$. From Libál, Reichhardt, and Olson Reichhardt, 2006.

$N_{i} / N$ of the $N$ vertices that are of the type $i$ as a function of colloidal charge $q_{e}$ in a system with fixed $\kappa$ and distance between the traps. At $q_{e}=0$, the fractions of the vertex types match what is expected in a noninteracting system, while as $q_{e}$ increases, type I and VI vertices disappear first followed by type II and IV vertices, until for $q_{e} \geq 2.0$ only the type III ice rule obeying vertices remain. Figure 3(b) illustrates the fraction of vertex types as a function of the distance $d$ between the traps in a system with fixed $q_{e}$ and $\kappa$. When $d$ is small, the colloids are strongly coupled and type III vertices dominate, while as $d$ increases, the vertex distribution gradually shifts back to the random configuration. The results in Fig. 3(b) are similar to the observations in the initial work on magnetic ASI, where increasing the spacing between magnetic islands produced a more random state (Wang et al., 2006). In a system with fixed $q_{e}$ and $d$ and changing screening strength $\kappa$, shown in Fig. 3(c), the interaction between the colloids is weak for strong screening and the vertex distribution becomes random. A sample with fixed $d$ and $\kappa$ at $q_{e}=0.4$ that is subjected to an additional biasing drive $F^{d c}$ at $45^{\circ}$ from the $x$ axis is initially in the type IV biased state. As $q_{e}$ increases, Fig. 3(d) shows that a transition occurs into the lower energy type III square ice state. Libál, Reichhardt, and Olson Reichhardt (2006) also found that a transition from an ordered ice state to a disordered state occurs as a function of increasing temperature. These results indicate that a particle-based artificial ice system exhibits the same ice rule obeying states as nanomagnetic ASIs, with the advantages of the mesoscopic character of the particles which makes the microscopic degrees of freedom readily accessible.

\section{Experimental realization}

The realization of a full optical system, following the original proposition (Libál, Reichhardt, and Olson Reichhardt, 2006), remains a challenging task because (a) it would require a large optical power to generate enough double wells, and (b) it is difficult to finely tune electrostatic interactions between colloidal particles. An alternative realization was demonstrated recently using a combination of different techniques including soft lithography, magnetic manipulation, and optical tweezers (Ortiz-Ambriz and Tierno, 2016). The experimental system, illustrated in Figs. 4(a) and 4(b), features interacting paramagnetic colloids confined by gravity in lithographically generated topographic double wells. Each trap contains two deep wells connected by a small central hill, and these indentations are arranged in a regular lattice, such as the square ice geometry illustrated in Fig. 4(a). Using optical tweezers, each trap is filled with one paramagnetic colloid consisting of a spherical polymer particle that is responsive to magnetic fields. Repulsive and tunable interactions are induced by applying an external magnetic field $\boldsymbol{B}=B \hat{z}$, perpendicular to the particle plane. The applied field induces a dipole moment $\boldsymbol{m}=\pi d^{3} \chi \boldsymbol{B} /\left(6 \mu_{0}\right)$ within the particles, where $d$ is the particle diameter, $\chi$ is the magnetic volume
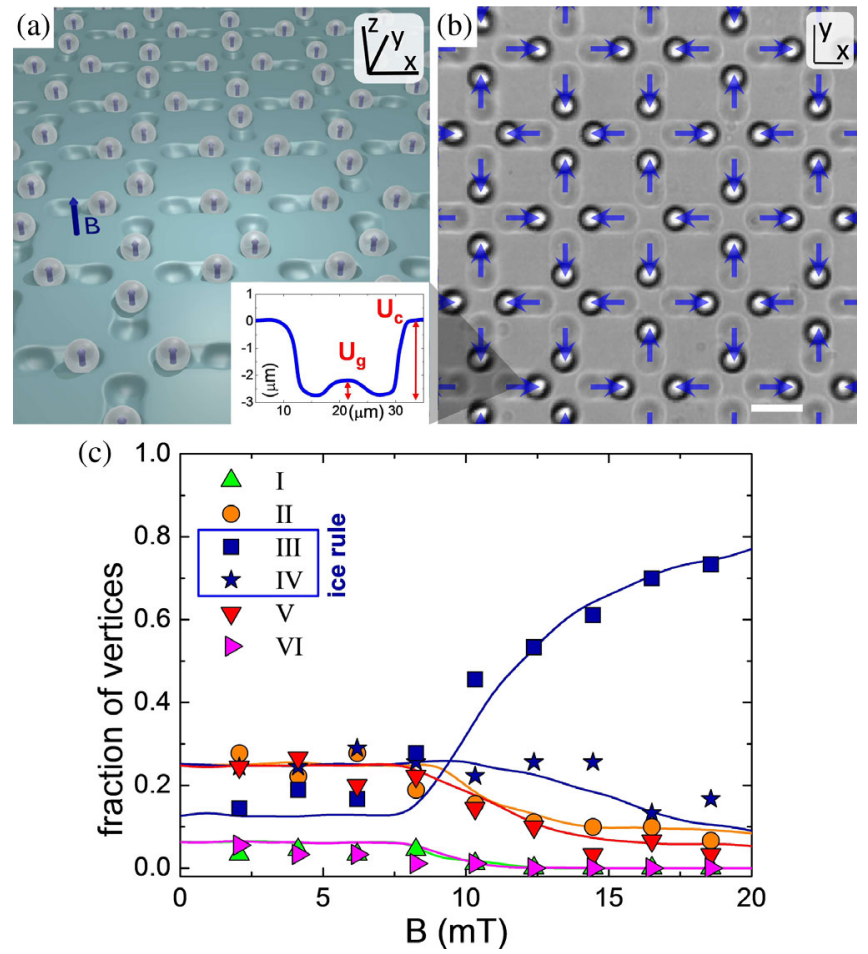

FIG. 4. (a) Schematic of a colloidal ice on a square lattice. Blue arrows in the particles are induced moments due to the applied field $\boldsymbol{B}$. Inset at the bottom shows a cross section of one double well obtained via confocal microscopy. From Loehr, OrtizAmbriz, and Tierno, 2016. (b) Microscope image of the experimental system in the $(\hat{x}, \hat{y})$ plane. Here the arrows indicate the spin associated with the particles, and the scale bar is $20 \mu \mathrm{m}$. (c) Fraction of vertices for square ice vs applied field. Experimental data are scattered points, and continuous lines are numerical simulations. Adapted from Ortiz-Ambriz and Tierno, 2016. 
susceptibility, and $\mu_{0}$ is the permeability of the medium (water). All particles interact through magnetic dipolar interactions. Pairs of particles $(i, j)$ with moments $\boldsymbol{m}_{i, j}=\boldsymbol{m e}_{i, j}$ and at distance $r=\left|\boldsymbol{r}_{i}-\boldsymbol{r}_{j}\right|$ interact through dipolar forces, with an interaction potential given by

$$
U_{d}(r)=\omega\left[\frac{\boldsymbol{e}_{i} \cdot \boldsymbol{e}_{j}}{r^{3}}-\frac{3\left(\boldsymbol{e}_{i} \cdot \boldsymbol{r}\right)\left(\boldsymbol{e}_{j} \cdot \boldsymbol{r}\right)}{r^{5}}\right],
$$

with $\omega=\mu_{0} m^{2} /(4 \pi)$. This potential is maximally attractive (repulsive) for particles with magnetic moments parallel (perpendicular) to $\boldsymbol{r}$. In an unconstrained system, when the applied field is perpendicular to the particle plane, $U_{d}$ reduces to an isotropic repulsion between particles confined on the same plane $U_{d}(r)=\omega / r^{3}$. The field amplitude is chosen such that a confined particle, when subjected to the dipolar force from a neighbor, can cross the central hill with gravitational potential $U_{g}$, but never escape from the bistable confinement $\left(U_{c}\right), U_{g}<U_{d}<U_{c}$, as shown in the inset in Fig. 4(a).

Following the original idea (Libál, Reichhardt, and Olson Reichhardt, 2006), one can assign a vector to each particle pointing toward the well occupied by the colloid; see Fig. 4(b). This mapping makes it possible to construct a set of vertex rules similar to ASIs. However, in contrast to ASI whose islands have in-plane dipole moments, the colloidal ice features out-of-plane dipoles, and thus the energetic hierarchy is similar to that found for repulsive electrostatic colloids. Within a homogeneous lattice, collective interactions between the particles oppose the local energetics and enforce the ice rule for the colloidal system (Nisoli, 2014). Indeed, systematic measurements combined with Brownian dynamics simulations confirm that, for high field amplitudes, the colloidal ice follows the ice rule (Ortiz-Ambriz and Tierno, 2016). The experiments were performed by setting the system in a random configuration using optical tweezers, turning on the magnetic interactions, and waiting for the system to equilibrate. The initial disordering process was necessary since, in contrast to the original simulations (Libál, Reichhardt, and Olson Reichhardt, 2006), the magnetic colloids experience negligible thermal fluctuations due to the relatively large particle size $d \sim 10 \mu \mathrm{m}$. The disordering process can be considered as a way to couple the system with a virtual heat bath, while increasing the strength of the pair interaction represents a cooling procedure. Moreover, in analogy with ASIs, the particle-based ice reaches a unique ground state (GS) filled by type III vertices. The loss of degeneracy results from the different particle distance at the vertex, which makes the type III and IV vertices energetically different. The most energetically unfavorable vertices with three (type V) or four (type VI) colloids in become topologically connected to the low-energy type II and I vertices, reducing their occurrence at large $\boldsymbol{B}$, as shown in Fig. 4(c).

\section{Defect dynamics, grain boundaries, and logic gate}

The experimental system provides a versatile approach for probing the effect of disorder and topological defects in frustrated lattices. The optical tweezers, which are independent from the collective magnetic coupling, can be used to manually add or remove particles from the double wells. One method for creating an artificial defect line is to start with the square ice GS (all type III vertices) and flip particles in a sequence that produces a double line of type IV vertices connecting two high-energy type II and V vertices. These defects can be described within the framework of the "dumbbell" model (Castelnovo, Moessner, and Sondhi, 2008). To each vertex one can associate an effective topological "charge" $q$ that quantifies the degree of violation of the ice rule. As explained in Sec. II.A, a spin pointing toward (away from) the vertex center will have a positive (negative) charge $q_{i}$, and the total charge at each vertex $i$ will be $q=\sum_{i} q_{i}$. Thus, the GS of the square lattice corresponds to $q=0$ (type III), whereas type II (V) vertices have a positive $q=+2$ (negative $q=-2$ ) charge; see Fig. 5(a). Using this formalism, it was shown that in a 3D spin ice and at low temperature topological defects interact only via a magnetic Coulomb law $V(r) \propto Q^{2} / r$, where $Q$ is the topological charge and $r$ is the separation distance between the two monopoles.
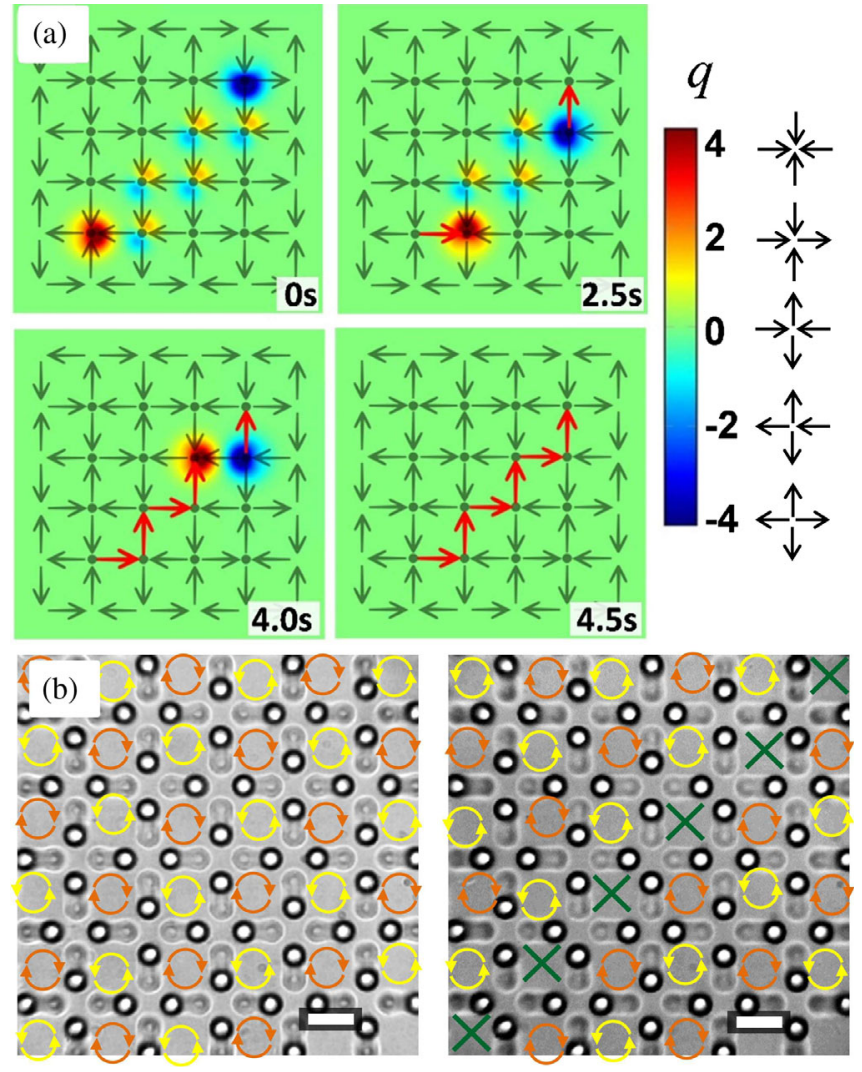

FIG. 5. (a) Color map showing the vertex charges for a line of flipped particles connecting two $q= \pm 2$ defects. These defects annihilate after $t=4.5 \mathrm{~s}$ due to the external field, applied at $t=0 \mathrm{~s}$. Adapted from Loehr, Ortiz-Ambriz, and Tierno, 2016. (b) Microscope images showing a square ice state prepared in its ground state of type IV $(q=0)$ vertices (left), or in a metastable state with a grain boundary (right). The square plaquettes can have a clockwise (orange [light gray] arrows) or counterclockwise (yellow [white] arrows) chirality (type IV vertices), or be achiral (green [gray] crosses). Scale bars are $20 \mu \mathrm{m}$ for both cases. Adapted from Ortiz-Ambriz and Tierno, 2016. 
However, numerical simulations demonstrated that for a 2D square ASI, such strings shrink due to an additional line tension term (Mól et al., 2009) that results from the missing degeneracy of this lattice at the single vertex level. In the 2D case, the interaction potential between two defects becomes $V(l)=-Q / l+v l+c$, where $v$ is the line tension and $c$ is a constant associated with the creation of defect pairs (Silva et al., 2013). Direct measurements of the average defect line length $\langle l\rangle$ showed that the defects shrink following overdamped dynamics described by

$$
\gamma \frac{d l}{d t}=-\frac{\partial V}{\partial l}=-\frac{Q}{l^{2}}-v,
$$

where $\gamma$ is a friction coefficient. Equation (4) lacks the inertial term that is present when describing the motion of topological defects in ASIs (Vedmedenko, 2016). This first order nonlinear equation admits as a solution the implicit function,

$t-t_{0}=\frac{1}{\beta}\left\{l_{0}-l+\sqrt{\alpha}\left[\arctan \left(\frac{r}{\sqrt{\alpha}}\right)-\arctan \left(\frac{l_{0}}{\sqrt{\alpha}}\right)\right]\right\}$,

where $\alpha=Q / v$ is the ratio between the Coulombic and line tension contributions. The solution describes a monotonous shrinkage of the defect line starting from $l_{0}$ at $t_{0}$, and was used to fit both the experiments and the simulation data. Such analysis makes it possible to quantify $\alpha=0.0290 \pm 0.0014 a^{2}$, where $a=29 \mu \mathrm{m}$ is the lattice constant of the square system. This Coulombic charge is 1 order of magnitude lower than the value calculated for magnetic ASI (Mól et al., 2009), indicating that the line tension contribution is enhanced in the colloidal ice. Nevertheless, two topological charges that are bound by a defect line either in ASIs or in the colloidal ice interact with a similar Coulombic law.

The modeling of the magnetic colloids is similar to that used for charged colloidal systems since the colloids are confined in double-well traps and experience a repulsive pairwise interaction with each other. The colloid dynamics is governed by an overdamped equation of motion given by

$$
\frac{1}{\mu} \frac{d \mathbf{r}_{i}}{d t}=\sqrt{\frac{2}{D d t}} k_{B} T N[0,1]+\mathbf{F}_{p p}^{i}+\mathbf{F}_{s}^{i},
$$

where $D$ is the diffusion constant, $\mu$ is the mobility, $d t$ is the time step, and $N[0,1]$ is a Gaussian distributed random number with a mean of zero and a variance of 1 . The first term is the thermal force. Magnetization of the colloids in the $z$ direction produces a repulsive particle-particle interaction force $\mathbf{F}_{p p}(r)=A_{c} \hat{\mathbf{r}} / r^{4}$ with $A_{c}=3 \times 10^{6} \chi^{2} V^{2} B^{2} / \pi \mu_{0}$ for colloids at a distance $r$ apart, where $V=\pi d^{3} / 6$ is the colloid volume. The substrate force $\mathbf{F}_{s}^{i}$ traps the colloids in the double wells. Simulations of magnetic colloids have focused on a range of parameters that matches what is used in the experiments.

Since it is possible to tune the interaction forces between the particles by changing the applied field, it is interesting to model the interaction between different types of monopoles in a square colloidal ice for increasing field amplitude. Loehr, Ortiz-Ambriz, and Tierno (2016) initialized the magnetic colloids in a square ice substrate in a GS containing two monopole excitations of opposite charge and separated by a distance $d$. These monopoles are attracted and approach each other at a velocity that increases with the applied magnetic field. In subsequent simulations Libál et al. (2017) showed that at high fields, the monopoles annihilate more rapidly through a nucleation process in which additional monopoles appear and break the defect string which extends between the original two monopoles. This work also showed that different monopole species move at different velocities, in contrast to what would be expected for magnetic spin ice systems. By applying a periodic biasing field, the asymmetry in the monopole mobilities can be exploited in order to create a ratcheting motion of a defect line.

It is also interesting to investigate grain boundaries (GBs) in the colloidal ice. In general, GBs are ubiquitous in condensed matter and they influence the performance of a variety of systems including high $T_{c}$ superconductors (Graser et al., 2010), organic films (Rivnay et al., 2009), and direct-band gap semiconductors (van der Zande et al., 2013). In the square colloidal ice, as in ASIs, GBs emerge as defect lines that separate unmatched regions of the GS; see Fig. 5(b). While GBs have been observed in magnetic samples (Morgan et al., 2011; Zhang et al., 2013), monitoring their formation and dynamics remains a challenging task due to the small length scales of the ASIs. In terms of plaquettes and not vertices, the GS of the square ice is composed of a checkboard pattern of loops with alternating chirality, indicated by yellow (white) and orange (light gray) in the left panel of Fig. 5(b). For the square ice this GS is twofold degenerate, and a $90^{\circ}$ rotation around a vertex produces another GS. A domain wall, shown in the right panel of Fig. 5(b), can be constructed by a line of achiral cells that separates two incompatible regions of GS. These are very stable defects because, in contrast to a double line of type IV vertices, it is necessary to modify all the vertices on one side of the domain wall to make them compatible with the GS of the other side. This feature could be used to store binary information. By assigning a value of 0 (1) to a clockwise (counterclockwise) chiral cell, it is possible to use a binary representation to write logical information directly in the GS of a lattice of colloidal ice or nanoscale ASI, and later erase the information using a strong field which restores the full GS (Ortiz-Ambriz and Tierno, 2016).

A major interest in nanoscale magnetism and ASIs is the realization of logic circuits based on a dense array of strongly magnetized elements. The particle-based ice could provide a guideline for the realization of simple and resettable logic ports based on magnetic dipolar interactions. Figure 6 shows a proposal for a "NOR" gate, a functionally complete port capable of generating all logical functions (Bartee, 1991). The gate is based on a metastable biased state, filled by highenergy type IV vertices, and it can be initialized and reset by an external force $\boldsymbol{F} \sim(\boldsymbol{B} \cdot \nabla \boldsymbol{B})$ applied along one diagonal $\boldsymbol{F}_{1}=F_{1}(\hat{\boldsymbol{y}}-\hat{\boldsymbol{x}})$. In this system, the colloids in two horizontal rows of traps have been replaced with particles that have a higher magnetic susceptibility (pink arrows in Fig. 6). A single particle in the upper left corner is used to trigger the propagation of a defect line. Then a second biasing force $F_{2}$ is applied to flip only the two chains containing high susceptibility particles. The input of the gate is applied by switching the line of colloids with higher susceptibility, such that a 
(a)

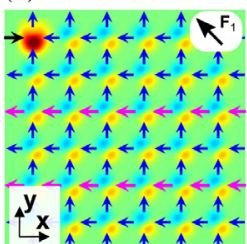

(b) (c) input-phase

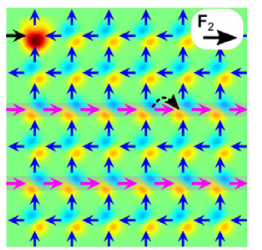

(d) output-phase

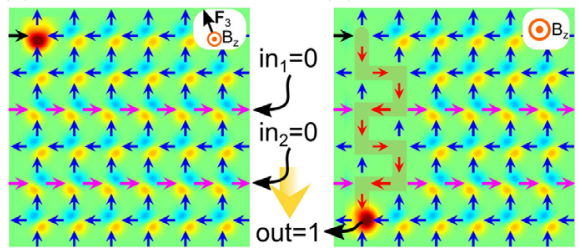

(e) input-phase

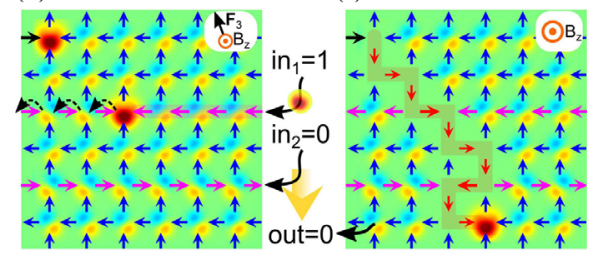

FIG. 6. Proposal of a NOR gate using the colloidal ice. (a), (b) System preparation: a force $F_{1}$ creates a biased state (a), while another force $F_{2}<F_{1}$ reverses the spins only along two rows of particles that have smaller susceptibility (b). (c), (d) Images showing a 1 output obtained from two $(0,0)$ inputs. The defect propagation is induced by a field $B$ applied perpendicular to the plane, while an additional small force $F_{3}$ applied along the diagonal of the ice lattice prevents the introduction of other defects from the upper left corner. The final result shown in (d) is the 1 output. (e), (f) Images showing a 0 output obtained from $(1,0)$ input. In (e) the 1 input of the first row containing particles with high susceptibility is induced by an extra charge coming from outside the region. From Loehr, Ortiz-Ambriz, and Tierno, 2016.

flipped line means (1) true and an unflipped line means (0) false. As shown in Figs. 6(c) and 6(d), the defect starts to propagate under a perpendicular field $\boldsymbol{B}=B \hat{z}$ and is deflected by the flipped input lines. After propagation, the location of the charge in the bottom row gives the output. If it is located in the lower left corner, the output is (1). If either of the two input lines are in a 1 state, the defect line will not be deflected, and the topological charge will end up in a different position, giving an output of (0).

\section{E. Effect of disorder, doping, and system memory}

A general open question in frustrated systems is how robust or fragile the frustrated states are in the presence of quenched disorder. Numerical simulations of spatially extended samples with significant statistics can shed light on this aspect. In the colloidal artificial ice, one method of introducing quenched disorder is by randomizing the energy of the barrier at the center of each double well. In a square ice geometry subjected to a biasing field, such randomness induces the formation of +1 or -1 monopoles in the background of biased ice rule obeying vertices. Libál, Reichhardt, and Olson Reichhardt (2012) studied the effect of quenched disorder on a colloidal square ice and found that under simulated annealing, instead of a completely ordered state, the system formed a partially disordered configuration containing monopoles and biased ice rule obeying vertices.

Under the application of a cyclic biasing drive, the monopole defects begin to annihilate until the sample reaches a reversible state in which the same effective spin configuration appears during each biasing cycle, so that the system exhibits what is known as return point memory (Pierce et al., 2005). Libál, Reichhardt, and Olson Reichhardt (2012) also showed that the return point memory state is reached much faster in a kagome colloidal ice than in a square colloidal ice. In the kagome ice, irreversibility is produced by the motion of individual defects, which can be pinned rapidly by the quenched disorder, whereas in the square ice, the irreversible motion arises from the reconfiguration of grain boundaries, and these extended objects require a longer time to find a pinned configuration.

Return point memory can be quantified by examining the overlap function $q_{o}$ which compares the effective spin configurations from one cycle to the next at the same biasing field,

$$
q_{o}\left(F_{\text {ext }}\right)=N^{-1} \sum_{i=1}^{N} S_{i}^{n-1}\left(F_{\text {ext }}\right) S^{(n)}\left(F_{\text {ext }}\right) .
$$

Here $S_{i}$ is an effective spin equal to $S_{i}=1\left(S_{i}=-1\right)$ if the colloid is sitting in the right or top end of the trap (left or bottom end), and $n$ is the number of cycles that the external force $F_{\text {ext }}$ is applied. When $q_{o}=1.0$, the spin configuration is perfectly reversible and is exactly the same during each biasing field cycle, while $q_{o}=-1.0$ would indicate that the spin configuration is exactly the opposite of its previous value. Figures 7(a) and 7(b) show $q_{o}$ vs $F_{\text {ext }}$ for the square and the kagome colloidal artificial ices containing random disorder in the barrier heights implemented using a Gaussian distribution of width $\delta$. The kagome system is characterized by a modified version of the ice rule with 2 -in/1-out or 1-in/2out vertex types. With repeated cycles of the biasing field, $q_{o}$
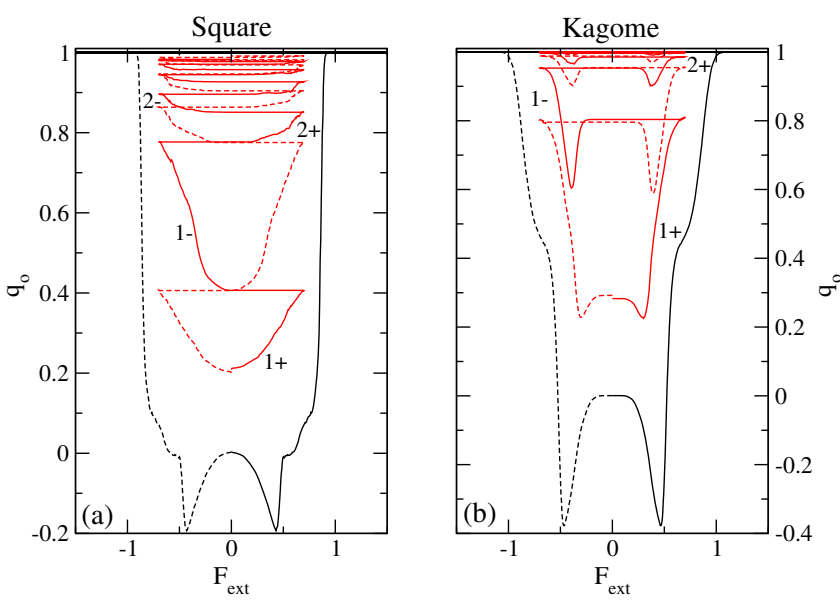

FIG. 7. Effective spin overlap $q_{o}$ vs biasing field $F_{\text {ext }}$ during consecutive hysteresis loops averaged over ten disorder realizations for (a) a square ice sample with disorder strength $\delta=0.1$ and (b) a kagome ice sample with $\delta=0.1$. Outer line: Saturated loop with $F_{\max }=2.0$, including the initial curve. Inner lines: Unsaturated loops with $F_{\max }=0.7$, with $n$ increasing from bottom to top; the first few half loops are labeled. Solid lines: clockwise loops; dashed lines: counterclockwise loops. In the kagome ice, $q_{o}$ approaches 1 after only a few cycles, while a much larger number of cycles are required before $q_{o} \approx 1$ in the square ice. From Libál, Reichhardt, and Olson Reichhardt, 2012. 
approaches 1.0, indicating the emergence of return point memory. This process takes about $n=10$ cycles in the square ice but only $n=4$ cycles in the kagome ice. The steady state arrangement of artificial spins is not ordered but contains numerous defects; however, the same pattern of defects appears after each biasing field cycle. Return point memory effects have also been observed in ASI by Gilbert et al. (2015). It would be interesting to understand how these effects appear in many of the other proposed ASI geometries (Morrison, Nelson, and Nisoli, 2013). Further modeling work on kagome and square colloidal artificial ices that took into account the effects of temperature showed that when biasing fields are present or when the system is prepared in different metastable states, a series of structural transitions can occur, and that states with monopole ordering appear (Olson Reichhardt, Libál, and Reichhardt, 2012).

In ASI, each spin is constrained to point along the axis of the magnetic nanoisland, although it is possible to remove entire islands. In particle-based artificial ices, it is possible to introduce doubly occupied sites, which would be like having spins pointing in both directions on the same nanoisland. Conversely, it is also possible to remove a particle to create a vacancy or dilution of the spin arrangement. Libál, Olson Reichhardt, and Reichhardt (2015) considered the square and kagome geometries containing doubly occupied wells where the system was initiated in a GS configuration and then subjected to an increasing temperature. The effects of the doping are very different in the two ice geometries, as shown in Fig. 8 where the doping ratio $x$ is held constant. For the square ice at low temperature, $N 3$ (3-in/1-out) vertices form around each doping site in order to screen the doubly occupied wells. As $T$ increases, $N 1$ defects and $N 2$ biased defects begin to appear near the doping sites. Thus in the square ice, the doping sites act like weak spots or nucleation points that generate increased hopping of the colloids in the surrounding sites. In contrast, the kagome ice GS can readily absorb the doubly occupied sites without creating monopole states, as shown in Fig. 8(d) at low temperature. This is because the kagome GS consists of an equal number of GS vertices $N 1_{g s}$ and $N 2_{g s}$, and the system can shift this balance so that there are slightly more $N 2_{g s}$ vertices, enabling it to incorporate the extra charge of the doubly occupied sites while still remaining in a GS configuration. As the temperature increases, the doped sites in the kagome lattice reduce rather than increase the amount of hopping occurring in the neighboring vertices.

\section{F. Kagome ice and its inner phase}

Kagome systems have been the subject of intense research in nanomagnetism because this simple geometry provides a truly degenerate frustrated system (Tanaka et al., 2006; Qi, Brintlinger, and Cumings, 2008). The degeneracy arises at the single vertex level, where the three spins have equal distances and interaction energies. The ice rule of this lattice is associated with a net topological charge, with $q=+1$ for 2-in/1-out and $q=-1$ for 1 -in/2-out. Although the kagome ice does not have a well-defined GS, it was shown theoretically in ASIs that the long range tail of magnetic dipolar interactions can further reduce the entropy and give rise to ordered phases. Specifically, multipole expansion (Möller and
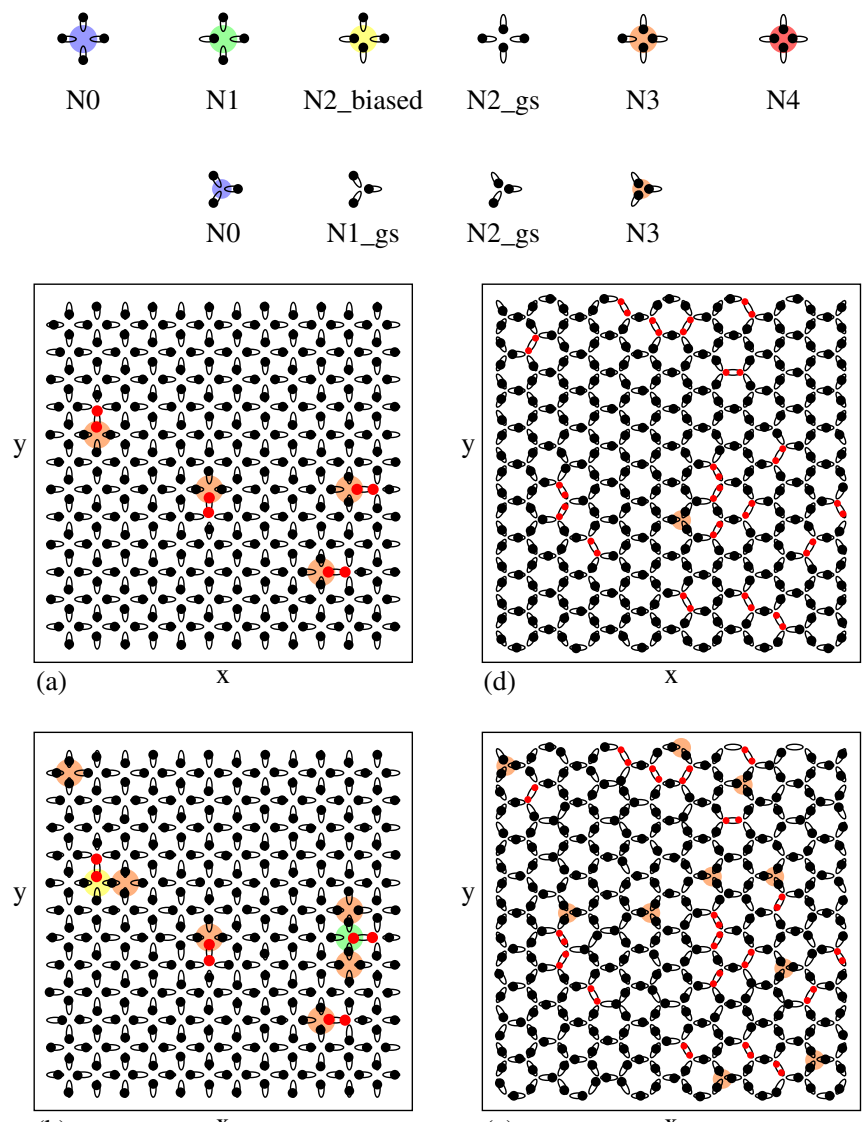

(b)

(e)

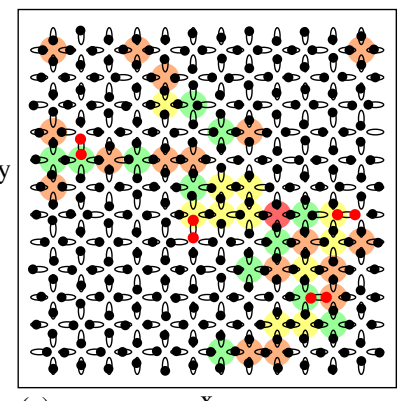

(c)

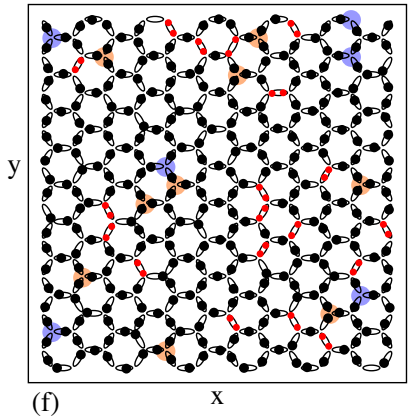

FIG. 8. Small portions of the square (left) and kagome (right) colloidal ice with double-well traps (open ellipses), colloids in singly occupied traps (filled black circles), and colloids in doubly occupied traps (filled red [gray] circles); the different vertex types are illustrated at the top of the figures. (a)-(c) The square ice system at a doping ratio of $x=0.0236$. (a) At $T=1.0$, below melting, each doped site is screened by an N3 monopole. (b) At $T=5.0$, some thermal wandering of the $N 3$ sites can occur, and $N 1$ states can form at the doped sites. (c) At $T=12$ the regions away from the doped sites remain in the ground state while local melting occurs at and near the doped sites. (e)-(g) The kagome ice system at a doping of $x=0.095$. (e) At $T=1.0$, below melting, the ground state absorbs the doping charge without forming defects by increasing the ratio of $N 2_{g s}$ to $N 1_{g s}$ vertices. (f) At $T=12.0, N 3$ monopoles form in regions away from the doped sites. (g) At $T=15.0, N 1$ monopoles begin to appear. From Libál, Olson Reichhardt, and Reichhardt, 2015.

Moessner, 2009) and numerical simulations (Chern, Mellado, and Tchernyshyov, 2011) predicted that lowering the temperature should favor the transition from a short-range ordered 
phase ("ice I") to a long range ordered state ("ice II"). In the latter case the spins on the hexagons define chiral and achiral loops along the lattice. Finally, the system can transition into a completely ordered "spin solid," or chiral phase, with chiral and achiral loops alternating in a chessboardlike pattern. Direct visualization of these exotic states with their associated relaxation dynamics remains elusive. An indirect signature of the ice I phase has been reported based on magnetotransport measurements of the Hall signal in a cooled ASI sample (Branford et al., 2012). Further, the ice II phase was visualised via MFM on a lattice of permalloy nanoislands heated above the Curie temperature of the constituent material (Zhang et al., 2013). More recently, low-energy muon spectroscopy was used to probe the existence of peaks in the muon relaxation rate that can be identified with a critical temperature associated with a phase transition that bridges such phases (Anghinolfi et al., 2015). Numerical simulations of the magnetic colloidal system in the kagome ice geometry show the emergence of different states as the colloid-colloid interaction strength changes (A. Libál et al., 2018). For weak interactions, the system is in a paramagnetic state, as shown in Fig. 9(a). As the interaction strength increases, the system first enters a state where the ice rule is obeyed in an ensemble of disordered spins, as illustrated in Fig. 9(b). This is followed by
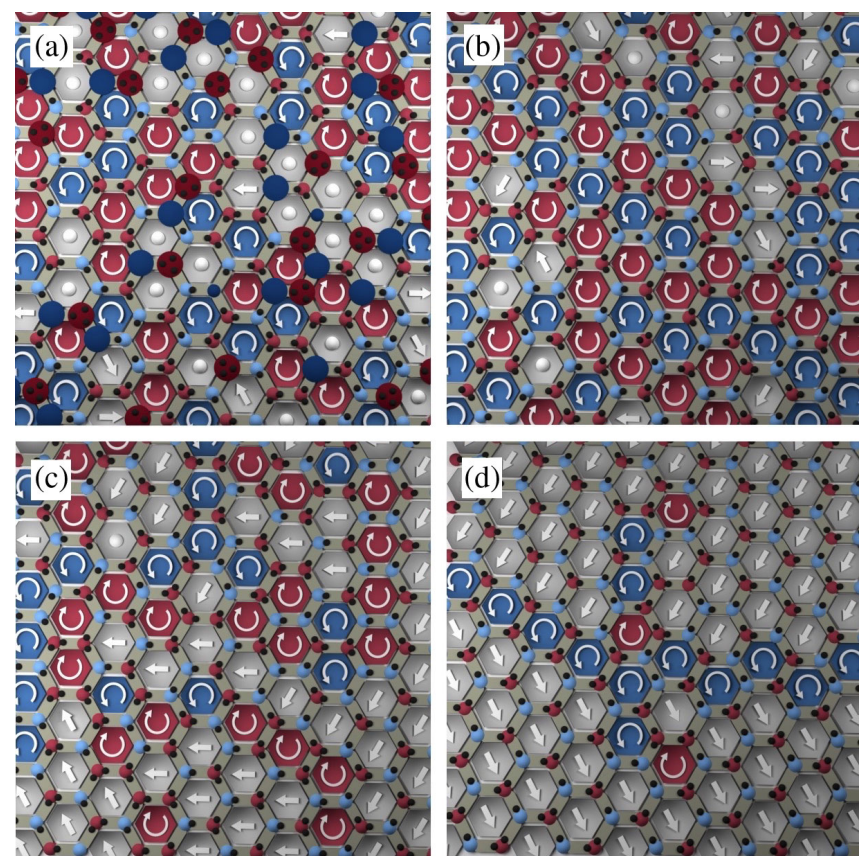

FIG. 9. Results from numerical simulations of the kagome particle-based ice. Each double-well trap (light gray) holds a single paramagnetic colloid (dark gray dots). The hexagonal plaquettes contain arrows indicating the plaquette chirality direction or effective biasing field $F_{b}$ for chiral and achiral plaquettes, respectively. Dots indicate that no $F_{b}$ value can be assigned. (a) Paramagnetic phase at $B=0 \mathrm{mT}$. Large disks with chiral arrows indicate $q_{n}= \pm 3$ vertices with $n=3$ and 0 , respectively. (b) Charge-free phase at $B=13.2 \mathrm{mT}$ containing only $q_{n}= \pm 1$ vertices. (c) Partially charge ordered phase at $B=$ $24 \mathrm{mT}$ with domains of charge and spin ordered vertices and plaquettes. (d) Ferromagnetic phase at $B=40 \mathrm{mT}$ containing a grain boundary. From A. Libál et al., 2018. the appearance of the topologically ordered charged state shown in Fig. 9(c), and finally, in the limit of very strong interactions, a threefold degenerate ferromagnetic state emerges as shown in Fig. 9(d). Unlike the phases predicted and experimentally observed with ASI, the colloidal kagome ice forms a ferromagnetic state at high interaction strength. This ferromagnetic state arises due to interactions between nonnearest neighbors, rather than simple vertex energetics, and disappears when the interactions are short ranged, explaining why it was not observed previously.

\section{G. Other geometries}

In addition to the square and kagome artificial ices, other types of frustrated systems have been realized with microscale colloids. Chern, Reichhardt, and Olson Reichhardt (2013) proposed a system of colloids interacting with a honeycomb array of optical traps that contain three wells instead of two; see Fig. 10(a). This system is a realization of a fully packed loop (FPL) model and Baxter's three-coloring problem (Baxter, 1970). As a function of temperature and interaction strength, a series of phases appears in the triple well system, including a stripe state, stripes with sliding symmetries, random packed loop states, and disordered states containing broken loops. Figure 10(b) illustrates the disordered state, while Figs. 10(c) and 10(d) show the random FPL states.

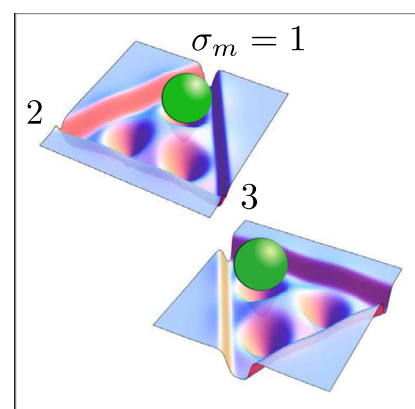

(a)

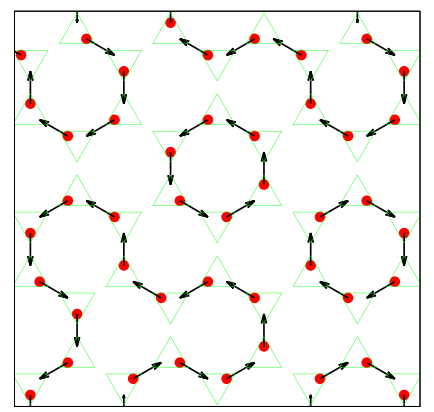

(c)

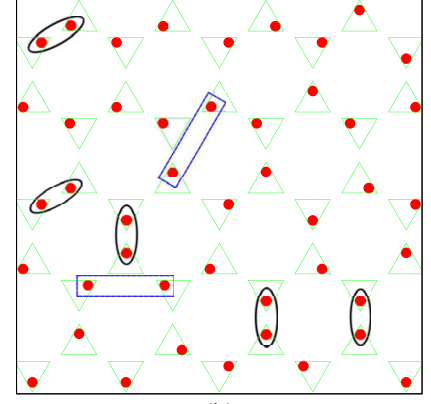

(b)

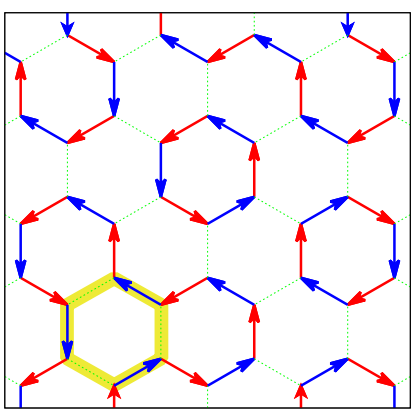

(d)
FIG. 10. (a) Schematic diagram of the basic unit cell with two triple well traps, each containing one colloidal particle; (b), (c) are snapshots of a small portion of the system. The green (gray) triangles represent the traps and the dots denote the particles. (b) Random distribution of particles at high temperatures; (c) an example of a particle configuration that can be mapped to random fully packed loops in the hexagonal lattice, as illustrated in (d). From Chern, Reichhardt, and Olson Reichhardt, 2013. 

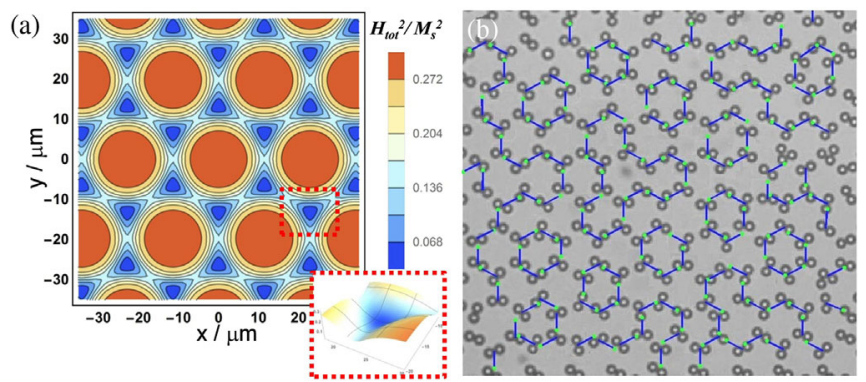

FIG. 11. (a) Magnetostatic energy landscape of a ferrite garnet film (FGF) calculated under a static, magnetic field perpendicular to the FGF $B=3.6 \mathrm{mT}$. The inset shows a $3 \mathrm{D}$ view of one triangular minimum. (b) Random FPL phase observed with a monolayer of paramagnetic colloids above the FGF after an annealing performed with a precessing magnetic field. From Tierno, 2016.

FPL models have been used to explain a broad class of phenomena in magnetism, optics, and polymer physics (Blöte and Nienhuis, 1994; Duplantier, 1998; O'Holleran et al., 2008; Jaubert, Haque, and Moessner, 2011), but physical realizations are rather scarce. An experimental realization of the colloidal version has been achieved using a honeycomb lattice of triangular shaped magnetic minima, as shown in Fig. 11(a). The complex magnetic potential arises when a uniaxial ferrite garnet film (FGF) is subjected to a constant magnetic field $\boldsymbol{B}_{z}=B \hat{\boldsymbol{z}}$. The FGF is composed of a triangular lattice of magnetic "bubbles," i.e., cylindrical ferromagnetic domains that are uniformly magnetized (Tierno, Johansen, and Fischer, 2007; Tierno et al., 2009). Paramagnetic colloids dispersed above this honeycomb magnetic lattice self-assemble into interacting microscopic dimers. To anneal the lattice into its minimum energy state, the system is subjected to a precessing magnetic field consisting of a combination of the perpendicular field and an in-plane rotating field $\boldsymbol{B}_{x y} \equiv B_{x y}[\cos (\omega t) \hat{\boldsymbol{x}}-\sin (\omega t) \hat{\boldsymbol{y}}]$. The resulting field $\boldsymbol{B}=$ $\boldsymbol{B}_{z}+\boldsymbol{B}_{x y}$ performs a conical precession around the $\hat{z}$ axis with angular frequency $\omega$, and sets the dimers into rotational motion. Depending on the field parameters, the final dimer arrangement after application of the field can be mapped to a long-range striped phase or to a random FPL state, as illustrated in Fig. 11(b). The mapping of the dimers to the FPL model is achieved by using the arrow representation originally introduced by Elser and Zeng (1993)) for the spin$1 / 2$ kagome antiferromagnet. Since the dimer sits on one of the three sides of a triangular minimum, an arrow can be defined that points from the dimer center to the free corner of the triangle. This is shown by a green dot with a blue line in Fig. 11(b). FPLs arise when arrows forming closed loops visit each lattice vertex only once.

Another recent realization of a particle ice composed by topographic double wells but in a different geometry is the highly coordinated triangular lattice $(z=6)$ presented by Lee and Tierno (2018). Although not directly inspired by the water or spin ice materials, the triangular geometry reflects the spin disposition in several magnetic materials with moments lying on weakly coupled parallel planes (Nakatsuji et al., 2005; Dublenych, 2017). The triangular order reflects the natural arrangement of repulsive particles in the absence of the substrate. However, such ordering can be frustrated by the presence of the central hill in the double wells. In this geometry, collective interactions between the particles lead to a unique GS characterized by vertices with three colloids pointing inward and three outward, similar to what was predicted for ASIs by Mól, Pereira, and Moura-Melo (2012) and Rodrigues et al. (2013). It was also found that the use of a bias force that magnetizes the system allows the GS to be accessed easily via a structural, martensiticlike transition characterized by the coherent sliding of one particle at each vertex.

\section{THE FRAGILE ICE MANIFOLD}

\section{A. Nature of the ice rule in colloidal ice}

While the ASI and the particle-based ice have often been considered as equivalent, their frustration and energetics are fundamentally different. At the nearest neighbor level, a vertex in the particle-based ice has the lowest energy unfrustrated configuration in which all particles are far from the vertex. Indeed the nearest neighbor energy of a magnetic spin ice vertex with $n$ spins pointing toward the vertex is proportional to the square of its topological charge,

$$
E_{n} \propto q_{n}^{2}
$$

thus favoring the ice rule, which minimizes the topological charge. ${ }^{1}$ In contrast, the energy of a vertex in particle-based ice scales as

$$
E_{n} \propto n(n-1)
$$

where $n(n-1)$ is the number of repulsive interactions among $n$ particles. This energy favors $n=0$ and 1 states, which correspond to large negative charges according to Eq. (1). In particular, the energies of the particle-based ice vertices do not possess a $\mathcal{Z}_{2}$ symmetry. Thus the local energetics actually work against the ice rule. The ice rule in particle-based ice is recovered only in the thermodynamic limit and its origin is collective: not all vertices can simultaneously have all particles located away from the vertex.

In a finite-size particle-based ice system of uniform coordination, the energy can be reduced by pushing particles onto the boundaries. The total charge in the bulk is then bounded by the flow of the pseudospin field through the boundary. Since the pseudospins have an absolute value of 1 , the density of charge in the bulk scales at least as the reciprocal of the length of the boundaries, leading to an ice manifold in the thermodynamic limit. In a system of multiple coordination, however, such as $z=4,3$, we can consider the $z=3$ vertices as an "internal boundary" onto which the $z=4$ sublattice can push topological charges. This leads to the emergence of negative charges on $z=4$ vertices, in violation of the ice rule. Note that this behavior, which was predicted (Nisoli, 2014; Nisoli, 2018b) and verified numerically and

\footnotetext{
${ }^{1}$ Here we neglect, for argument, the known geometric effects that can lift the degeneracy in the square geometry.
} 
experimentally (András Libál et al., 2018), points to an essential difference in the origin of the ice rule in magnetic and particle-based spin ices. The ice rule in magnetic ice is known to be robust against decimation (Morrison, Nelson, and Nisoli, 2013), mixed coordination (Gilbert et al., 2014, 2016), and dislocations (Drisko, Marsh, and Cumings, 2017), and is present even in finite sized clusters (Li et al., 2010). In particle-based ice, however, the local energy in Eq. (9) opposes the ice rule, which is regained only as a collective compromise. Other differences appear in the kinetics. For example, when defect lines in square colloidal ice are driven with a field, the two monopoles at the ends of each line have different mobilities since, unlike the monopoles in magnetic spin ice, they have different energies (Libál et al., 2017).

The similarities and differences of the magnetic and particle-based ices can be quantified exactly. A mean field approach provides useful quantitative predictions that are fit well by experimental and numerical results (Nisoli, 2014; Libál et al., 2017). Starting with the approximation of a gas of decorrelated vertices, a constraint must be introduced so that the total topological charge is conserved. This takes the form of a Lagrange multiplier $\phi$ that modifies the original vertex energies from those in Eq. (9) to

$$
\tilde{E}_{n}=E_{n}-q_{n} \phi \text {. }
$$

For a lattice of coordination $z$, the choice $\bar{\phi} \sim(z-1)$ ensures conservation of topological charge, giving spin-ice-like effective energetics $\tilde{E}_{n} \sim q_{n}^{2}$, and therefore a spin ice behavior. Equation (10) can be interpreted as follows: the collective effect of the particle-sharing vertices can be subsumed into a emergent field $\phi$, which modifies the energetics of the individual vertex. Indeed, in a better approximation, one can allow the constraint-enforcing field $\phi(x)=\bar{\phi}+\eta(x)$ to fluctuate in space, since it mediates an entropic interaction among the topological charges to which it is coupled. This results in a familiar Debye picture for purely entropic screening with a correlation length $\xi^{2} \sim T / \overline{Q^{2}}$, where $\overline{Q^{2}}$ is the charge fluctuation of the manifold. For instance, in a fully degenerate square ice, $\overline{Q^{2}}=0$ in the ice manifold and thus the correlation length is infinite, as mentioned in the section on the topological properties of the ice rule. In contrast, $\overline{Q^{2}} \geq 1$ in kagome ice since each vertex has a charge of at least \pm 1 and the ice phase is never critical.

Consider now a lattice of multiple coordination. It is impossible to find a value of $\phi$ that can return a $\mathcal{Z}_{2}$ invariant, icelike effective energetics in Eq. (10) for both sublattices. One of the lattices must break the ice rule. How this happens can be understood by a geometrically intuitive, exact treatment (Nisoli, 2018b). The energy of the system is given by

$$
H=\sum_{\mathbf{y} \neq \mathbf{y}^{\prime}} \psi\left(\left|\mathbf{y}-\mathbf{y}^{\prime}\right|\right)
$$

where $\psi(r)$ is an isotropic repulsive interaction and $\mathbf{y}$ labels the position of the particles in the traps. These positions represent a binary variable that we can represent as - or - - Indeed Eq. (11) does not look like a spin ice Hamiltonian. Then we can ascribe a positive charge to the

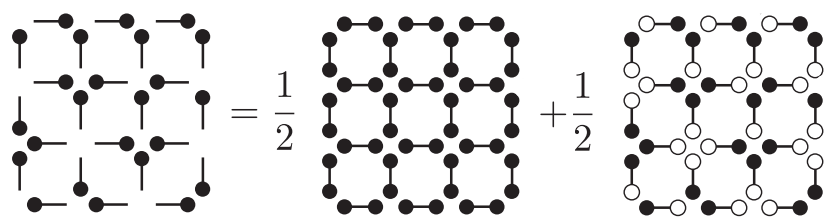

FIG. 12. Schematics showing that a colloidal ice, here in a random configuration, can be decomposed into a spin ice and a background exerting a geometric field. From Nisoli, 2018b

real particles and we can consider the empty locations $\mathbf{y}^{-}$of the traps as virtual negative charges $\bigcirc$, which repel (attract) other negative (positive) charges. We can fractionalize a trap on an edge $\mathbf{x}$ as

$$
-\mathbf{O}=\frac{1}{2} \bullet+\frac{1}{2} \mathrm{O}-\mathbf{\bullet}
$$

i.e., a positive dumbbell - (a trap doubly occupied by positive charges) plus a dipole of negative and positive charges represented by a spin $\vec{\sigma}=\bigcirc-\longrightarrow$ located in $\mathbf{x}$, the center of the trap. Then the energy in Eq. (11) can be rewritten as

$$
H=\frac{1}{2} \sum_{\mathbf{x} \neq \mathbf{x}^{\prime}} \sigma_{\mathbf{x}}^{i} J_{i i^{\prime}}\left(\mathbf{x}-\mathbf{x}^{\prime}\right) \sigma_{\mathbf{x}^{\prime}}^{i^{\prime}}-\sum_{\mathbf{x}} \vec{\sigma}_{\mathbf{x}} \cdot \vec{B}(\mathbf{x})
$$

The first term is the interaction between dumbbells and is clearly a spin ice Hamiltonian. $J_{i i^{\prime}}(\mathbf{x})$ is a tensor field and the background field $\vec{B}$ mediates the interaction between dipoles and the positive dumbbells, both of which can be reconstructed from the particle-particle repulsive interaction $\psi$. Figure 12 illustrates this decomposition. It follows that a particle-based ice becomes equivalent to a magnetic spin ice when the second term in Eq. (13) is zero. That is certainly true if a lattice has point reflection symmetry in the middle points $\{\mathbf{x}\}$ of each edge and explains why the kagome and square particle-based ices follow the ice rule, as found previously numerically and experimentally (Libál, Reichhardt, and Olson Reichhardt, 2006; Loehr, Ortiz-Ambriz, and Tierno, 2016; Ortiz-Ambriz and Tierno, 2016).

\section{B. Decimated systems}

The analogy between the colloidal ice and nanoscale ASI does not hold in general for more complex geometries. Imagine decimating a simple lattice such as the kagome by removing some traps. This breaks the reflection symmetry and the background field will perturb the spin ice energetics since the saturated dumbbells are replaced by negatively saturated ones at the locations of the missing traps. As shown in Fig. 13, the decimated system is equivalent to a spin ice stuffed with negative charges. The charges polarize the dumbbells close to the decimated vertices, breaking the ice rule. In these geometries with mixed coordination $z$, the conservation of the topological charge holds only at the global level, not at the sublattice level.

In the case shown in Fig. 14(a), the square system $(z=4)$ is decimated with optical tweezers to a lattice of mixed coordination $z=3,4$. The decimation process is equivalent to a 


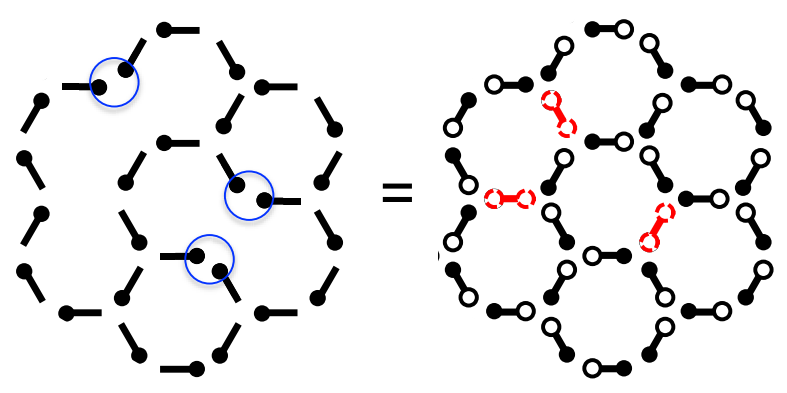

FIG. 13. Schematics showing that a decimated particle-based ice obtained by removing traps from a regular lattice is equivalent to a spin ice stuffed with negative charges at the locations of the missing traps. This leads to violations of the ice rule through formation of positive topological charges [blue (gray) circles]. From Nisoli, 2018b.

partial, random dimer covering of the edges, as illustrated in Fig. 14(b). Randomly chosen edges of the square lattice are covered by dimers under the constraint that each vertex contains at most one dimer. Removing an edge between two "dimerized" vertices with $z=4$ gives rise to only vertices of $z=3$. The process avoids formation of $z=2$ vertices and thus simplifies the vertex hierarchy of the mixed coordination system (Gilbert et al., 2014), although similar effects can also be observed in the presence of $z=2$ vertices.

Combined experiments and numerical simulations show that, in such a geometry, the ice rules are spontaneously yet selectively violated via the formation of negative topological monopoles of charge $q=2$ on the vertices of high coordination $z=4$. The low coordinated vertices $z=3$ still (a)

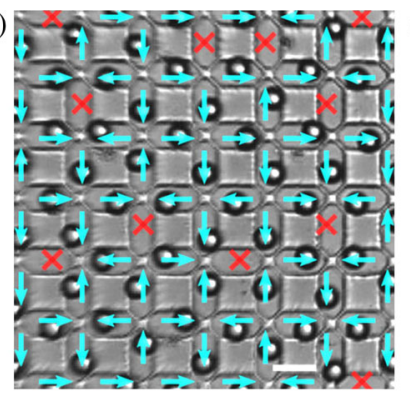

(c)

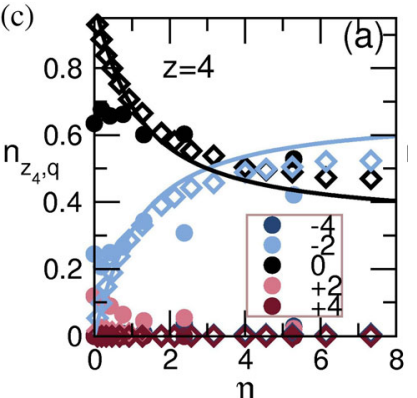

(b)
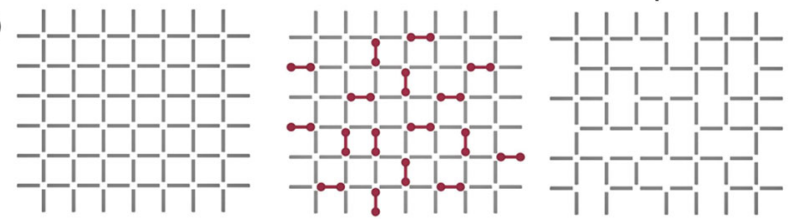

FIG. 14. (a) A decimated colloidal square ice where the missing spins (particles) are denoted by crossed arrows. (b) Decimation procedure for the square lattice that creates only vertices of coordination $z=3$ and 4 , but not $z=2$. This process is equivalent to a partial dimer covering [red (gray) dumbbells] of the edges. (c) Fraction of vertices $n_{z_{4, q}}$ vs decimation ratio $\eta=N_{z_{3}} / N_{z_{4}}$ for vertices with coordination number $z=4$ and at different values of the topological charge $q$. The experimental results (bullets) and numerical results (diamonds) are compared to theoretical predictions (solid lines). Adapted from András Libál et al., 2018. obey the ice rule (2-in/1-out or 2-out/1-in); however, the relative ratio of $q=1$ to $q=-1$ charges changes in order to compensate the negative charges that accumulate around the $z=4$ vertices. Even in this situation, the total topological charge of a system of "dipoles" remains zero.

A quantitative analysis of the experimental and theoretical results provides additional insight into the decimation process. Figure 14(c) shows the relative fraction of $z=4$ vertices, $n_{z_{4, q}}$, versus the ratio between the two vertex coordinations $\eta=N_{z_{3}} / N_{z_{4}}$, grouped by topological charge. The number of negative $q=-2$ charges generated around the $z=4$ vertices increases with increasing $\eta$, quantifying the strength of the ice rule violation. Above a critical decimation threshold, the entire system disorders due to the spontaneous appearance of entropydriven negative monopoles which induce topological charge transfer between the sublattices. As a result, the colloidal ice has a "fragile" low-energy manifold that is produced by an energetic compromise between locally excited vertices. This is in contrast to magnetic ASI, which are structurally "robust" ices. These observations prompt different exciting ideas. Since ice rule fragility is associated with topological charge transfer among sublattices, these new phenomena can be exploited for domain wall engineering, in which membranes that are semipermeable to the topological charge of defects are structurally designed. These results also apply to lattices of different coordination or to ASI systems of nonzero residual entropy.

\section{Discrete versus continuum models}

The interactions between the pseudospins in the colloidal ice are given by a generalized dipolar potential constructed from the effective interaction $V(r)$ between pairs of colloids (which in the case of magnetic colloids is $V \sim r^{-3}$ ). This implies that colloids with different types of repulsive interactions exhibit different phases within their ice manifold, and, in particular, that isotropically repulsive colloids are expected to more faithfully reproduce the phases of magnetic spin ices. Inspired by these results, in a theoretical study, Le Cunuder et al. (2019) investigated the energetics and phase transitions that occur in the kagome geometry by varying the temperature rather than increasing the interaction strength. Numerical calculations of the total magnetostatic energy for a system containing $N$ particles showed that the chiral state ("spin solid") always has a lower energy than the ferromagnetic one, a result which is robust regardless of the system size; see Fig. 15. This calculation was performed by assuming that the particles are fixed at the bottom of each trap, without considering any relative displacement.

The apparent discrepancy between the analytic results and those obtained in the simulations and experiments previously described was resolved by performing two types of Monte Carlo simulations (Le Cunuder et al., 2019). In the first discrete model, particles are allowed only to jump from one side of the bistable traps to the other, while in the second continuous model, particles are allowed to make these same jumps and also to move continuously around the lowest point of the bistable traps. While the discrete simulations reproduced the well-known phase structure of the dipolar spin ice, the continuous Monte Carlo simulations showed a single 


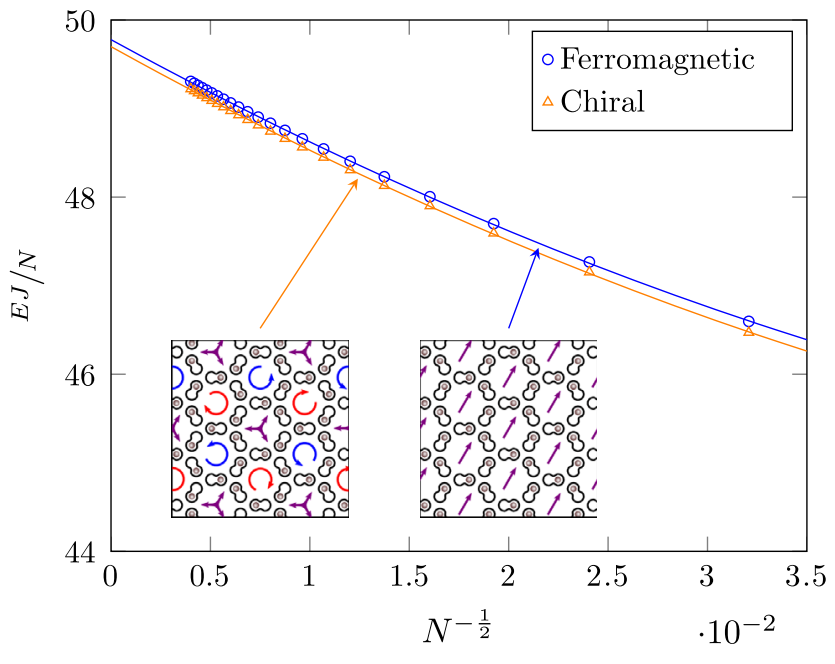

FIG. 15. Magnetostatic energy for $N=3 L^{2}$ interacting particles with periodic boundary conditions for the chiral [red (light gray) triangles] and ferromagnetic [blue (gray) circles] phases of the kagome ice (up to $L=144$ ). The inset shows schematics illustrating the two competing orders. Scattered points are numerical calculations and continuous lines are polynomial fits of the form $E / J \sim \alpha N+\beta N^{1 / 2}+\gamma$, where $\alpha$ is the energy per spin in the thermodynamics limit and $\beta$ and $\gamma$ are finite-size corrections. From Le Cunuder et al., 2019.

phase transition from charge disorder directly to the ferromagnetic state. Furthermore, using the continuous model, it was shown that by modifying the strength of the traps, the GS of the particle-based ice can be directly changed from the chiral ordering to the ferromagnetic one.

\section{Future directions in particle ice}

The experimental realization of particle ice represents a starting point for exploring the novel physics that emerges from the mesoscopic character of the colloidal system. A variety of avenues for future research are now available.

Relaxation and dynamics: Apart from the annihilation of simple defects, the kinetic mechanisms behind the relaxation dynamics of the particle ice remain largely unexplored. Since direct visualization of the dynamics is possible in colloidal ice, correlation functions for relatively long times can be extracted from the particle positions, and collective or local rearrangement events (spin flips) can be identified as the system ages from a metastable state. An interesting question is whether the colloidal ice could exhibit glassy behavior and kinetic arrest. The presence of disorder in the system, such as an inhomogeneous distribution of the hills within the double wells, or induced decimation, may make the energy landscape more rugged and induce glassy behavior. There have been reports of evidence of glassiness in frustrated systems from both theoretical models and experiments. For example, a spin glass phase was predicted to occur for a decimated square ice in an ASI (Sen and Moessner, 2015), and experiments with buckled colloidal monolayers (Zhou et al., 2017) showed evidence of dynamical arrest.

Thermalization effects: Using smaller particles with large thermal fluctuations would allow spontaneous particle switching within the double wells, a feature that is currently absent in the experimental system. This platform could be used to explore thermalization effects in the colloidal ice. Such an investigation could be performed by using optical tweezers to prepare a square or kagome ice lattice in the lowest energy state while keeping the particle-particle interaction strength high. Subsequently lowering the magnetic field would then favor thermal disordering starting from the GS and make it possible to determine the lifetime, fraction, and dynamics of the emerging, thermally induced "Dirac" strings.

Complex geometries: The lithographic approach imposes no limit on the type of two-dimensional structures that can be engineered. While most of the works on particle-based artificial ices have focused on square or kagome lattices, numerous other geometries that have been proposed for ASI (Morrison, Nelson, and Nisoli, 2013) could also be realized in particle-based systems. Since the particle ice system minimizes the global energy rather than the local vertex energy, it often exhibits fragility (András Libál et al., 2018), so it is likely that these systems could have very different behaviors than their ASI counterparts. In addition, aperiodic structures or even disordered lattices with hyperuniform properties can easily be designed and implemented with the colloidal ice.

Annealing procedures: Frustrated systems can easily be trapped in a metastable state when the material or sample is cooled to reach the GS. Different annealing protocols have been developed to date, such as thermalization during sample growth (Morgan et al., 2011) or above the Curie temperature of the constituent material (Zhang et al., 2013), and rotational demagnetization (Nisoli et al., 2007; Ke et al., 2008; Wang et al., 2018). However, most of the theoretically predicted ordered phases in highly frustrated systems are still far from being realized since these techniques do not allow for direct system visualization during annealing, making it impossible to monitor in situ how close or far from the GS the system is as a function of time. The colloidal ice could provide a way to directly follow the annealing process in situ. The current realization relies on static fields; however, it would be possible to introduce a time dependent field that spins the particles in order to induce tunable anisotropic or even time-averaged attractive in-plane interactions.

Three dimensions: A major effort of the frustrated magnetism community is devoted to the realization of a 3D artificial version of the pyrochlore lattice, such as by using magnetic wires or nanobars oriented at determined angles in order to replicate the tetragonal order (Fernández-Pacheco et al., 2017). An alternative approach to this goal was proposed by Mistonov et al. (2013) based on the realization of an inverted colloidal opal filled with cobalt via electrochemical crystallization. This opal was obtained using the colloidal crystal template technique, which is a well-established method in material science (Zakhidov et al., 1998), based on replicating the long-range order of an assembled colloidal crystal in a solid matrix. Although this type of approach may restrict the lattice geometry (Chern, Reichhardt, and Nisoli, 2014) or lead to artifacts due to domain wall pinning within the magnetic network, it represents a rather simple, fast, and versatile method of obtaining a highly ordered 3D porous structure. Future attempts at the realization of a 3D colloidal ice system may exploit similar self-assembly techniques. On the other 
hand, progress in optical manipulation has made it possible to create 3D optical traps for colloids (Grier and Roichman, 2006), and thus it should be possible to create new types of fully 3D spin ice geometries using optically confined colloids. In principle, such geometries could be used to model water ice more accurately or possibly as a method for realizing deconfined phases.

\section{OTHER PARTICLE-BASED FRUSTRATED SYSTEMS}

Frustrated configurations have also been realized for colloidal systems in the absence of a topographic substrate (Han et al., 2008). For example, when colloids confined to a 2D plane are allowed to buckle into the third dimension, as shown in Fig. 16, frustration emerges since upward and downward buckling are equal energy states. In the triangular lattice naturally formed by the colloids, the buckling process produces a structure similar to an antiferromagnetic Ising model on a triangular lattice (Shokef et al., 2013), and interesting disordered and stripelike patterns appear. While
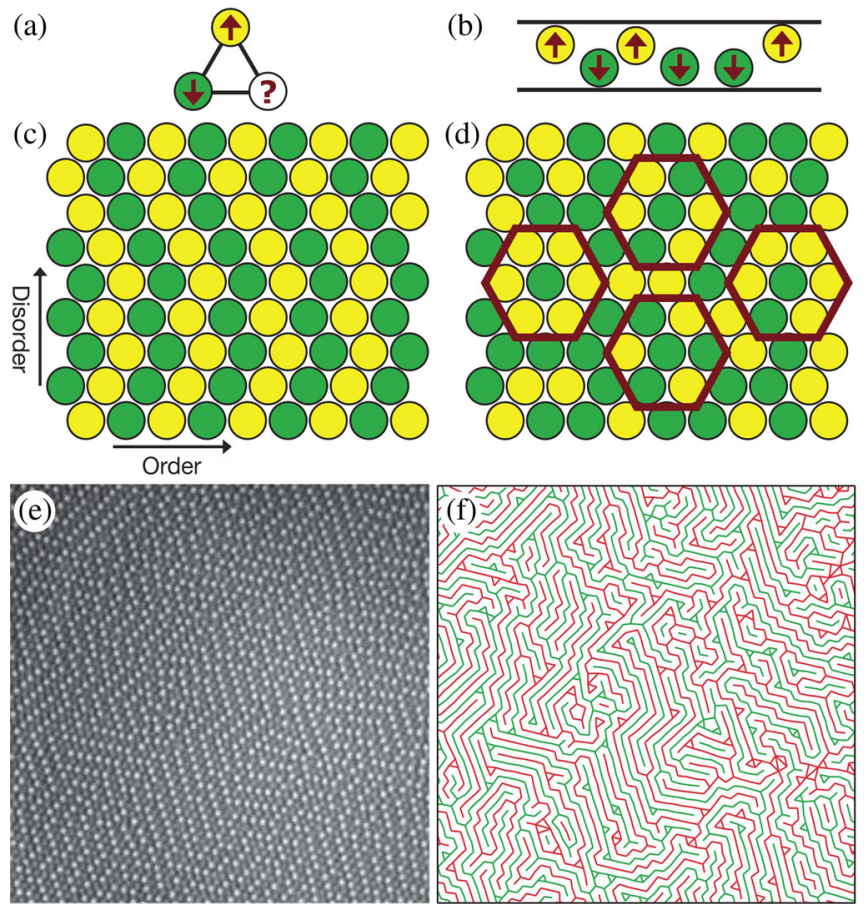

FIG. 16. (a) Three spins on a triangular plaquette cannot simultaneously satisfy all antiferromagnetic interactions. (b) For colloids confined between walls separated by a distance of the order 1.5 sphere diameters (side view), particles move to opposite walls in order to maximize their free volume. (c), (d) Ising-like GS configurations in which each triangular plaquette has two satisfied bonds and one frustrated bond. (c) Zigzag stripes generated by stacking rows of alternating up and down particles with random sideways shifts, where all particles have exactly two frustrated neighbors. (d) Particles in disordered configurations have $0,1,2$, or 3 frustrated neighbors [red (gray) hexagons]. (e) Microscope image of a buckled system where bright (dark) particles are close to the upper (lower) plane. (f) Corresponding labyrinth patterns with the frustrated up-up (down-down) bonds drawn in red (light gray) [green (gray)]. Adapted from Han et al., 2008. the original Ising antiferromagnet at low temperature features extensive entropy (Wannier, 1950; Wannier, 1973), the buckled system displays a subextensive one, which points toward the presence of glassy dynamics and kinetic arrest. Indeed glassiness in such a system was recently reported (Zhou et al., 2017), with other features such as the emergence of an order by disorder transition (Shokef, Souslov, and Lubensky, 2011; Leoni and Shokef, 2017). Other colloidal systems that can exhibit frustration effects include either particles with complex shapes (Brown, Smith, and Rennie, 2000) or isotropic ones deposited above deformed surfaces (Bausch et al., 2003; Soni, Gómez, and Irvine, 2018). In the first case, the frustration can be tuned by designing different shapes which compete with the confinement and impede crystallization, often producing a disordered state (Zhao and Mason, 2009, 2015). Tuning the shape of the confining surface may also lead to frustration effects between isotropically repulsive colloids. The surface topology will induce the formation of lattice defects such as disclinations, releasing energetic stresses arising from the packing on the curved confinement (Nelson, 2002; Irvine and Vitelli, 2012; Guerra et al., 2018).

On a macroscopic scale, geometric frustration has been addressed by arranging classical bar magnets. One significant case is shown in Fig. 17 where an ensemble of interacting millimeter-sized magnets is arranged into a kagome lattice. The basic units of the system are ferromagnetic rods attached to planar rational units which allow only out-of-plane angular motion (polar angle $\alpha$ ) but not in-plane motion (the angle $\theta$ is fixed). The initial state of the system was prepared by polarizing these "macroscopic spins" along the perpendicular plane $(\hat{z})$ via a strong static field. Switching off the field induces a relatively fast ( $\sim 2 \mathrm{~s})$ reorganization process, and the magnets stabilized into a equilibrium pattern filled by vertices with 2 -in/1-out and 1-in/2-out. The rotors relaxation process occurred in three steps; namely, a relatively fast inertial reorientation where the rotors break the axial symmetry, then a sequence of dissipative librations followed by final damped oscillations which lead to a nearest neighbor spin correlation $\left\langle\boldsymbol{s}_{i} \cdot \boldsymbol{s}_{j}\right\rangle=1 / 3$. Mellado, Concha, and Mahadevan, 2012 also showed the exciting possibility to extend the system toward 3D by stacking different rotors in a tetrahedral-like configuration. Such a demonstration manifests the ubiquitous (a)

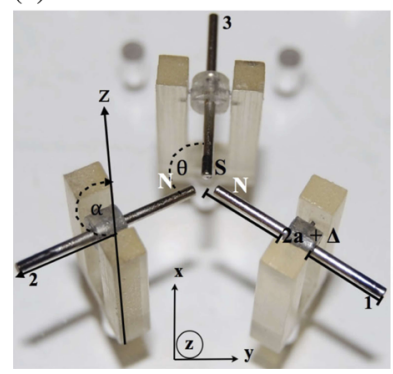

(b)

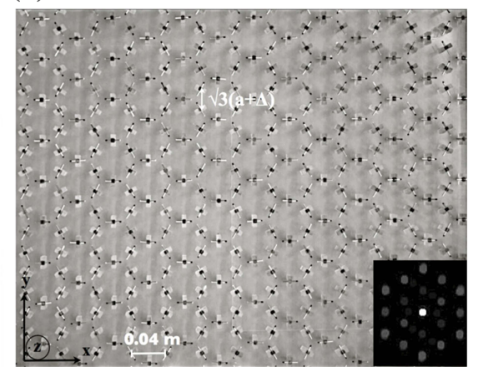

FIG. 17. (a) Three magnetic rotors composed of macroscopic ferromagnetic rods arranged at $\theta=120^{\circ}$ with respect to each other. (b) Macrosopic honeycomb lattice of rotors (inset shows the corresponding Fourier transform). From Mellado, Concha, and Mahadevan, 2012. 
character of geometric frustration, which transcends the length scale.

Numerous other condensed matter systems can be described as an assembly of particles with repulsive pairwise interactions. Examples include vortices in type II superconductors (Libál, Olson Reichhardt, and Reichhardt, 2009), ions (Pyka et al., 2013), dusty plasmas (Morfill and Ivlev, 2009), skyrmions (Ma et al., 2016), and Wigner crystals (Reichhardt et al., 2001). Any of these systems, when coupled to the correct substrate geometry, could exhibit effective spin degrees of freedom and the ice rule.

One of the first proposals for such particle-based artificial ices involved vortices in type II superconductors (Libál, Olson Reichhardt, and Reichhardt, 2009), where considerable progress was already made in creating various types of pinning arrays to control the vortex ordering. In the vortex system, a series of double-well traps can be fabricated by placing two pinning sites very close together, as illustrated in the top panels of Fig. 18. When the sample is nanostructured in this fashion, the thinner parts of the superconductor have lower vortex condensation energy, and the vortex will preferentially sit at the
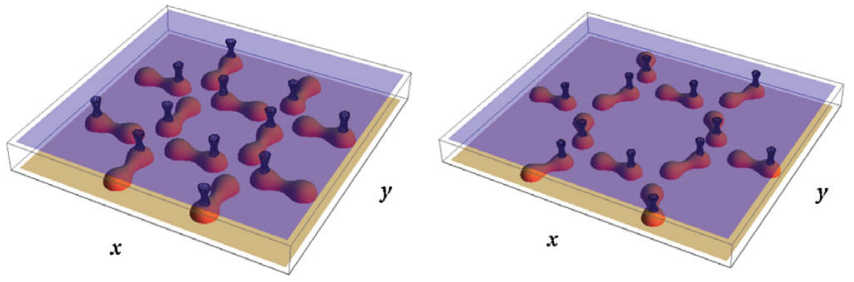

(a)
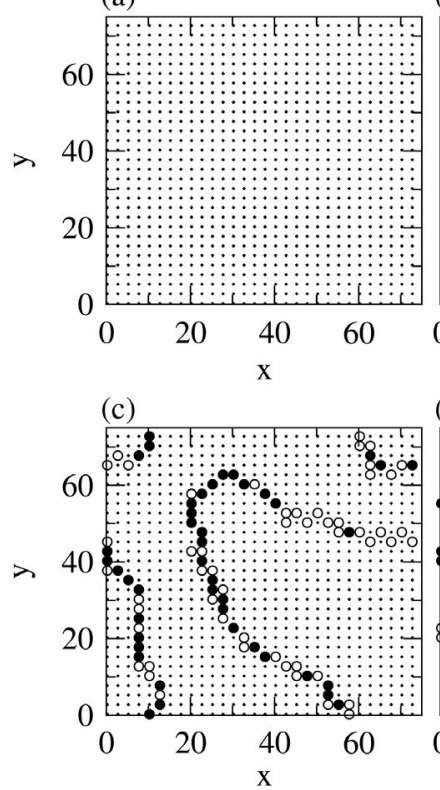

(b)

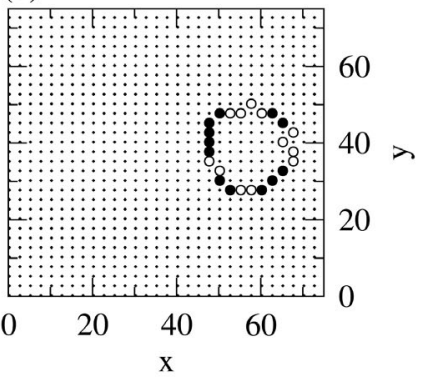

(d)

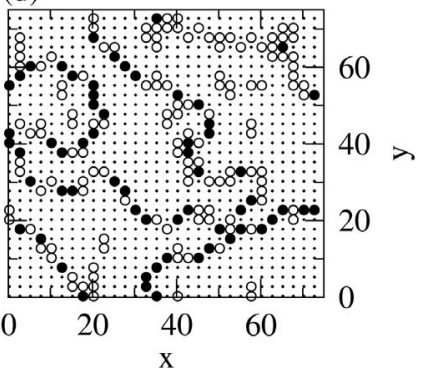

FIG. 18. Top panels: Schematic of the nanostructured pinning site configurations producing ice states. Double-lobed objects: pins; open mesh objects: vortices. Left: Square ice ground state. Right: One possible biased ground state of the kagome ice system. Grain boundary images in square ice samples for increasing disorder strength $\delta$. Dots: ground state $n_{\text {in }}=2$ ice rule obeying vertices; filled black circles: ice rule defects; white circles: nonice rule defects. (a) $\delta=0$, (b) $\delta=0.1$, (c) $\delta=0.5$, and (d) $\delta=1.0$. From Libál, Olson Reichhardt, and Reichhardt, 2009. highest points of the double-well traps. For $N_{p}$ double-well traps, the number of vortices $N_{v}$ is directly proportional to the magnetic field, so that when $N_{v} / N_{p}=1 / 2$, the system is equivalent to the colloidal square ice at half filling. Particlebased modeling of vortices in pinning arrays is performed using similar techniques as those described for the colloidal systems; however, the pairwise interaction between the vortices is a modified Bessel function and has a $\ln (r)$ form in thin film superconductors. Many superconducting samples contain a significant amount of intrinsic random disorder. Thus, Libál, Olson Reichhardt, and Reichhardt (2009) explored the effects of additional quenched disorder on the GS in a square ice pinning arrangement at $N_{v} / N_{p}=1 / 2$; see Fig. 18. As the disorder strength $\delta$ increases, a large number of vertices not obeying the ice rule appear in the GS in the form of grain boundaries, but individual monopoles that are not associated with a grain boundary do not begin to appear until $\delta \geq 0.5$. The appearance of defects arranged in grain boundaries was subsequently observed experimentally in a square ASI (Morgan et al., 2011).

The superconducting vortex configurations can be observed directly using imaging techniques, but it is also possible to probe the stability of such configurations by applying a current in order to depin the vortices. When the vortex arrangement is highly ordered, the depinning threshold or critical current $I_{c}$ is higher than when the vortices are disordered. Latimer et al. (2013) studied the critical currents of superconductors with square ice geometries in experiments. They found a series of peaks in the critical current $I_{c}$ as a function of vortex density. When the temperature is lowered, a prominent critical current peak appears at $N_{v} / N_{p}=1 / 2$, as shown in Fig. 19 which displays $I_{c}$ vs $H / H_{1}$, where $H_{1}$ is the first matching field. The inset shows a simulation of the flux configuration at the $f=1 / 2$ filling where the square ice rule obeying state is highlighted. For $H / H_{1}>1.0$, the vortices start to become doubly quantized, and another peak in the critical current appears at $H / H_{1}=3 / 2$, which again corresponds to an ice rule obeying GS.

In a series of experiments, Trastoy et al. (2014, 2015) examined spin ice pinning arrays for the high temperature superconductor made of ytterbium barium copper oxide, where thermal effects are important. They found that changes in resistance correspond to vortex configurations that are dominated by a geometrically frustrated energy landscape that favors icelike ordering and frustration, and they found a series of peaks in the transport response similar to what is found in ordered arrangements above square pinning lattice.

For vortices on a kagome lattice of pinning sites, various ordered and disordered arrangements have been observed in imaging experiments, and it has been argued that the long-range vortex-vortex interactions are insufficient to lift the degeneracy of the different vortex configurations in the strong pinning regime (Xue et al., 2017). As the magnetic field is increased, additional vortices become trapped in the interstitial regions surrounding the pinned vortices, which could be a step toward the realization of a stuffed artificial spin ice (Xue et al., 2018). Imaging experiments for vortex configurations on the kagome ice (Wang et al., 2018) showed the predicted kagome ice rule obeying states (Libál, Olson Reichhardt, and Reichhardt, 2009) 


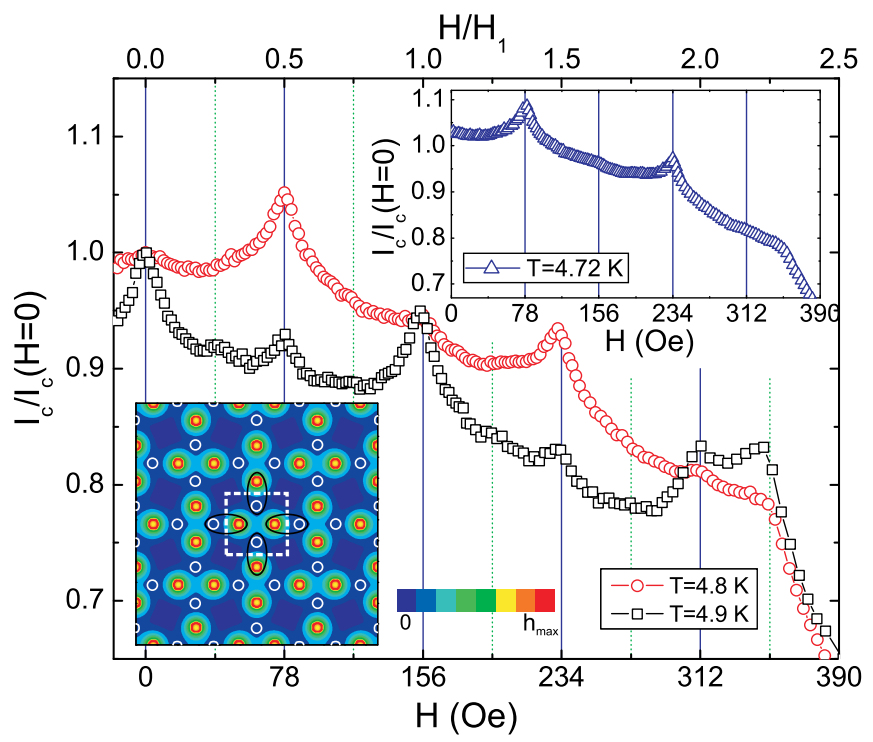

FIG. 19. The critical current of the vortex artificial square ice sample, normalized by the zero magnetic field critical current $I_{c}(0)$, as a function of the magnetic field $H$ at $T=4.8 \mathrm{~K}$ (red circles) and $T=4.9 \mathrm{~K}$ (black squares). The top inset shows the results obtained at $T=4.72 \mathrm{~K}$. The zero magnetic field critical currents are $I_{c}(0)=802 \mu \mathrm{A}$ at $T=4.72 \mathrm{~K}, I_{c}(0)=613 \mu \mathrm{A}$ at $T=4.8 \mathrm{~K}$, and $I_{c}(0)=335 \mu \mathrm{A}$ at $T=4.9 \mathrm{~K}$. The lower inset shows contour plots of the simulated local magnetic field $h$ for the half matching field, which shows the ground state vortex configuration at $T=4.8 \mathrm{~K}$. White circles indicate the positions of vortex-free holes and the dashed red square shows the unit cell of the simulation area. From Latimer et al., 2013.

both at $H / H_{1}=1 / 2$, as expected, and also at the higher field of $H / H_{1}=3 / 2$, where the additional vortices occupy the interstitial regions between pinning sites. Interestingly, the ice rule state at $H / H_{1}=3 / 2$ is even more ordered than the state at $H / H_{1}=1 / 2$, suggesting that the interstitial vortices may play a role in annealing defects in the ice GS.

Wang et al. (2016) examined vortex ordering and dynamics on a switchable artificial spin ice array. In this system, frustration effects can be switched on or off by changing the magnetic-charge ordering of the underlying reconfigurable magnetic ice substrate. Different magnetic-charge orderings can produce disordered or frustrated vortex configurations, as well as nonfrustrated configurations that produce ordered vortex crystals, as illustrated in Fig. 20 for different possible ordered states at $B / B_{\phi}=1.0$ and the $f=2.0$ filling. Here types I, II, and III correspond to different magnetic-charge ordered states of the magnetic substrate (Wang et al., 2018). Other work on vortex states with rewritable magnetic ice substrates showed that it was possible to switch between different patterns using an applied current (Ge et al., 2018).

Square and kagome spin ice geometries have also been proposed for skyrmions in magnetic dot arrays, where the dots are arranged such that the skyrmion can sit on either side of the trap. A recent work demonstrated that, when the skyrmions are strongly localized or particlelike, the behavior of the system is very similar to what is observed in both superconducting vortex and colloidal artificial ices (Ma et al., 2016). When the magnetic field is varied, however, the

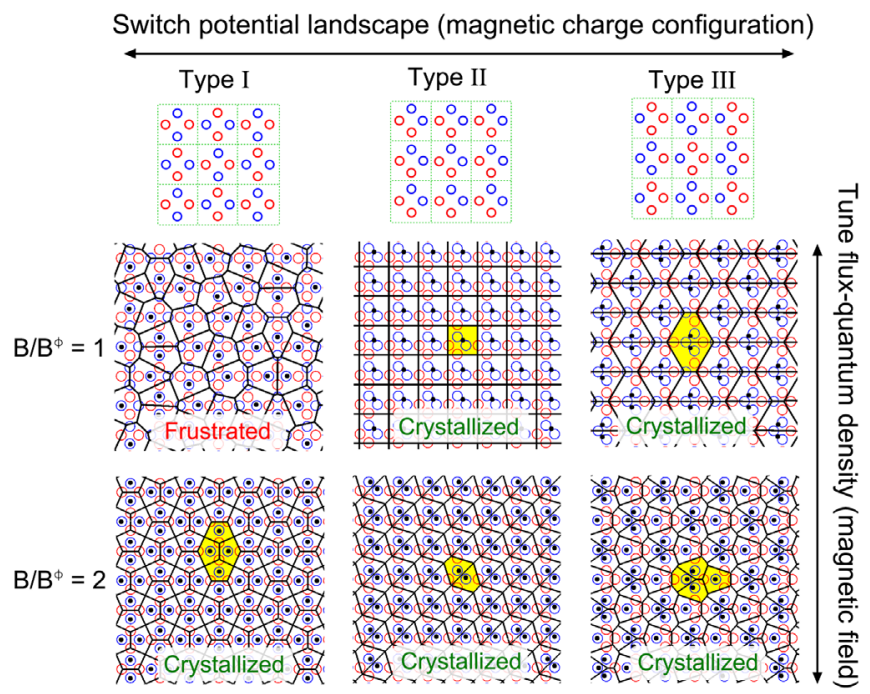

FIG. 20. Simulated distributions of flux quanta under types I, II, and III magnetic-charge orders and various flux-quantum densities. Solid black lines indicate Voronoi diagrams clarifying the flux-quantum ordering. One repeating unit structure in each of the crystallized flux-quantum lattices is highlighted in yellow. The disordered flux quanta under the type I magnetic-charge configuration at $B / B_{\phi}=1$ originate from geometric frustration with very high degeneracy. From Wang et al., 2018.

skyrmions can become elongated and fill the entire dot, destroying the effect that frustration has on the system. Thus, it can exhibit a transition from an ice rule obeying state to a sample with square or triangular ordered states.

\section{OUTLOOK}

In this section we provide further general directions that cover different disciplines across condensed matter systems not limited to particle-based systems.

Stuffed spin ice: In particle-based artificial ices, additional particles can be placed in the spaces between the traps of the substrate as well as within the traps themselves. These interstitial particles could potentially modify the ice rule for the particles that are sitting in the trap sites. One study has already provided evidence that the interstitial particles can actually enhance the ordering of the ice state (Xue et al., 2018). It would also be interesting to explore the dynamics of interstitial particles for different ice substrate configurations. For example, an ordered square ice background might produce increased or decreased diffusion of the interstitial particles compared to a background made of a degenerate GS ice.

Driven dynamics: In ASI the spin degrees of freedom are permanently localized on the magnetic nanoisland. In contrast, in particle-based artificial ices it is possible for the particles to hop from one ice substrate trap to another, making it possible to explore the dynamics of a frustrated system compared to that of a nonfrustrated one. Protocols that could be considered include sliding dynamics under a dc drive, shaken dynamics under external forces, or simply applying a drive to only one side of the sample but not to the other. This would be equivalent to subjecting a portion of an ASI to a magnetic field while the other portion of the sample experiences no field. 
Dynamic substrates: If optical traps are used to create the ice substrate, then a new type of dynamics involving the traps themselves could be implemented. For example, the trap could be flashed on and off, or the orientation of the doublewell potential could be rotated. Such protocols could provide new methods to reduce frustration effects. Alternatively, it may be possible to induce new types of frustration effects using dynamical protocols that bring repulsive particles together. Such protocols would create local high-energy states, but due to the frustration, there may not be an easy direction in which one of the particles could move away in order to reduce the interaction energy. In particle-based systems, dynamic protocols could be introduced readily by using dynamic traps (Curtis, Koss, and Grier, 2002; Bhebhe et al., 2018).

Frustrated active matter systems: Active matter composed of self-propelled particles is a rapidly growing research field with a rising number of experimental realizations of artificially self-propelled colloids (Bechinger et al., 2016). An interesting area for future exploration is frustrated systems on lattices where the fluctuations are active rather than thermal. Some studies have already shown the organization of active matter into vortex states (Beppu et al., 2017) and vortex lattices (Wioland et al., 2016; Nishiguchi et al., 2018), and it would be possible to arrange these vortices into a frustrated configuration. Additionally, if active and passive particles are mixed together, a frustrated ice geometry might reduce the diffusion of the active particles, while an ordered ice state could produce flow paths for the active particles. Other systems such as coupled gyroscopes or spinners could be placed in frustrated geometries which could lead to more complex dynamics (Nash et al., 2015).

Deformable substrates: In most of the ASI studied to date, the substrate or confinement is fixed and cannot react to the forces exerted on it by the particles. In soft matter systems there are many ways to create a substrate that could react and be deformed elastically. If this reaction is introduced into an ASI system, it could lead to new methods for reducing frustration effects. In addition, excitations such as monopoles could create additional long-range strain fields in the soft substrate that could be attractive or repulsive for other monopoles, leading to emergent effects such as excitons and polarons.

Nonlocal frustration effects: Numerous experiments have shown that large numbers of colloids can be addressed individually in optical feedback experiments (Lavergne et al., 2019). Thus, another route for studying frustrated systems would be to introduce nonlocal effects in which the forces experienced by particles in one part of the system are correlated with the positions of other particles that are far away. This would make it possible to test whether phase transitions can be induced by increasing the strength of the nonlocal interactions, to study the effects of small world versus random interactions, and to determine whether nonlocal effects change the nonequilibrium properties of the frustrated state. It would also be possible to engineer nonlocal frustration effects in which a reduction of frustration at one location in the system increases the frustration at a distant point, and to study how the system would relieve such effects.

Continuous spin directions: Another avenue in 2D soft mater systems is to allow the effective spin degree of freedom to point along multiple directions or have some freedom to move in the third dimension in order to create a frustrated Potts model, Heisenberg-like spin models, and/or $X Y$ models.

Nondissipative dynamics: In the particle-based models considered so far, the motion of the particles is overdamped. It is, however, possible to realize particle ices in which nondissipative effects are important, such as in underdamped systems including dusty plasmas, trapped ion crystals, or even millimetric magnets on 3D printed substrates. The inertial effects could produce phononic excitations, and it would be possible to study the difference in the phonon modes for crystalline versus degenerate or frustrated ice states, as well as the propagation of solitons or shock waves.

Functionalized colloids for frustrated geometries: There are many examples of functionalized colloidal systems in which the interactions between the colloids can be tailored to be anisotropic (Glotzer and Solomon, 2007), reconfigurable (Ortiz et al., 2014), or patchy (Bianchi, Blaak, and Likos, 2011), or even to mimic chemical bonds (Wang et al., 2012). Using such systems, it would be possible to create 2D or 3D colloidal assemblies that naturally exhibit frustration (Hynninen et al., 2007), or even a colloidal system that mimics the structure of water ice (Pauling, 1935).

Quantum effects: A final potential avenue would be to place cold atoms or cold ions on trap arrays arranged in an ice structure. This system could be realized at a size scale and temperatures for which quantum effects could become important (Glaetzle et al., 2015; Mazurenko et al., 2017). The transition from classical to quantum effects in frustrated systems may lead to new physical phenomena in a continuous evolving, yet mature field of research.

\section{ACKNOWLEDGMENTS}

We acknowledge helpful discussions with S. T. Bromley, B. Canals, J. Casademunt, L. Cugliandolo, T. M. Fischer, G. Franzese, S. Giblin, S. Klapp, D. Levis, A. Libal, H. Löwen, E. Oguz, J. Ortín, I. Pagonabarraga, N. Rougemaille, F. Sagués, J. M. Sancho, Y. Shokef, A. Snezhko, A. Straube, E. Vives, Z. Xiao, and W. Yong-Lei. A. O. A. and P. T. acknowledge support from the ERC Consolidator grant "ENFORCE" (No. 811234). The work of C. R., C. J. O. R., and C.N. was carried out under the auspices of the U.S. Department of Energy through the Los Alamos National Laboratory. Los Alamos National Laboratory is operated by Triad National Security, LLC, for the National Nuclear Security Administration of the U.S. Department of Energy (Contract No. 892333218NCA000001). P. T. acknowledges support from MINECO (ERC2018-092827), DURSI (2017SGR1061), and Generalitat de Catalunya under Program "ICREA Académia."

\section{REFERENCES}

Agra, R., F. van Wijland, and E. Trizac, 2004, "Theory of orientational ordering in colloidal molecular crystals," Phys. Rev. Lett. 93, 018304.

Anghinolfi, L., H. Luetkens, J. Perron, M. G. Flokstra, O. Sendetskyi, A. Suter, T. Prokscha, P. M. Derlet, S. L. Lee, and L. J. Heyderman, 
2015, "Thermodynamic phase transitions in a frustrated magnetic metamaterial," Nat. Commun. 6, 8278.

Arnalds, Unnar B., Jonathan Chico, Henry Stopfel, Vassilios Kapaklis, Oliver Bärenbold, Marc A. Verschuuren, Ulrike Wolff, Volker Neu, Anders Bergman, and Björgvin Hjörvarsson, 2016, "A new look on the two-dimensional Ising model: Thermal artificial spins," New J. Phys. 18, 023008.

Babič, Dušan, and Clemens Bechinger, 2005, "Noise-enhanced performance of ratchet cellular automata," Phys. Rev. Lett. 94, 148303.

Bader, S. D., 2006, “Opportunities in nanomagnetism,” Rev. Mod. Phys. 78, 1-15.

Baert, M., V. V. Metlushko, R. Jonckheere, V. V. Moshchalkov, and Y. Bruynseraede, 1995, "Composite flux-line lattices stabilized in superconducting films by a regular array of artificial defects," Phys. Rev. Lett. 74, 3269-3272.

Barrows, F., V. Brajuskovic, Amanda K. Petford-Long, and C. Phatak, 2019, "Emergent magnetic ordering and topological frustration in quasicrystal artificial spin ices," Phys. Rev. B 99, 094424.

Bartee, T.C., 1991, Computer Architecture and Logic Design (McGraw-Hill, New York).

Bausch, A. R., M. J. Bowick, A. Cacciuto, A. D. Dinsmore, M. F. Hsu, D. R. Nelson, M. G. Nikolaides, A. Travesset, and D. A. Weitz, 2003, "Grain boundary scars and spherical crystallography," Science 299, 1716-1718.

Baxter, R. J., 1970, “Colorings of a Hexagonal Lattice,” J. Math. Phys. (N.Y.) 11, 784-789.

Baxter, R. J., 1982, Exactly Solved Models in Statistical Mechanics (Academic, New York)

Bechinger, C., M. Brunner, and P. Leiderer, 2001, "Phase Behavior of Two-Dimensional Colloidal Systems in the Presence of Periodic Light Fields," Phys. Rev. Lett. 86, 930-933.

Bechinger, Clemens, Roberto Di Leonardo, Hartmut Löwen, Charles Reichhardt, Giorgio Volpe, and Giovanni Volpe, 2016, "Active particles in complex and crowded environments," Rev. Mod. Phys. 88, 045006.

Beppu, Kazusa, Ziane Izri, Jun Gohya, Kanta Eto, Masatoshi Ichikawa, and Yusuke T. Maeda, 2017, "Geometry-driven collective ordering of bacterial vortices," Soft Matter 13, 5038-5043.

Bernal, J. D., and R. E. Fowler, 1933, "theory of water and ionic solution, with particular refefence to $\mathrm{H}+$ and $\mathrm{OH}-$ ions," J. Chem. Phys. 1, 515.

Bhebhe, Nkosiphile, Peter A.C. Williams, Carmelo RosalesGuzmán, Valeria Rodriguez-Fajardo, and Andrew Forbes, 2018, "A vector holographic optical trap," Sci. Rep. 8, 17387.

Bianchi, Emanuela, Ronald Blaak, and Christos N. Likos, 2011, "Patchy colloids: state of the art and perspectives," Phys. Chem. Chem. Phys. 13, 6397.

Blöte, H. W. J., and B. Nienhuis, 1994, "Fully packed loop model on the honeycomb lattice," Phys. Rev. Lett. 72, 1372-1375.

Bohlein, Thomas, Jules Mikhael, and Clemens Bechinger, 2012, "Observation of kinks and antikinks in colloidal monolayers driven across ordered surfaces," Nat. Mater. 11, 126-130.

Bramwell, S. T., and M. J. P. Gingras, 2001, "Spin ice state in frustrated magnetic pyrochlore materials," Science 294, 1495-1501.

Branford, W. R., S. Ladak, Daniel E. Read, K. Zeissler, and L. F. Cohen, 2012, "Emerging Chirality in Artificial Spin Ice," Science 335, 1597-1600.

Brazda, T., A. Silva, N. Manini, A. Vanossi, R. Guerra, E. Tosatti, and C. Bechinger, 2018, "Experimental observation of the aubry transition in two-dimensional colloidal monolayers," Phys. Rev. X 8, 011050
Brown, A. B. D., C. G. Smith, and A. R. Rennie, 2000, "Fabricating colloidal particles with photolithography and their interactions at an air-water interface," Phys. Rev. E 62, 951-960.

Brunner, Matthias, and Clemens Bechinger, 2002, "Phase behavior of colloidal molecular crystals on triangular light lattices," Phys. Rev. Lett. 88, 248302.

Budrikis, Zoe, J. P. Morgan, J. Akerman, A. Stein, Paolo Politi, S. Langridge, C. H. Marrows, and R. L. Stamps, 2012, "Disorder Strength and Field-Driven Ground State Domain Formation in Artificial Spin Ice: Experiment, Simulation, and Theory," Phys. Rev. Lett.109, 037203; 110, 089902(E) (2013).

Budrikis, Zoe, Paolo Politi, and R. L. Stamps, 2011, "Diversity enabling equilibration: Disorder and the ground state in artificial spin ice," Phys. Rev. Lett. 107, 217204.

Canals, Benjamin, Ioan-Augustin Chioar, Van-Dai Nguyen, Michel Hehn, Daniel Lacour, François Montaigne, Andrea Locatelli, Tevfik Onur Menteş, Benito Santos Burgos, and Nicolas Rougemaille, 2016, "Fragmentation of magnetism in artificial kagome dipolar spin ice," Nat. Commun. 7, 11446.

Castelnovo, C., R. Moessner, and S. L. Sondhi, 2008, "Magnetic monopoles in spin ice," Nature (London) 451, 42-45.

Castelnovo, C., R. Moessner, and S. L. Sondhi, 2012, "Spin ice, fractionalization, and topological order," Annu. Rev. Condens. Matter Phys. 3, 35-55.

Chaplin, Martin, 2006, "Do we underestimate the importance of water in cell biology?," Nat. Rev. Mol. Cell Biol. 7, 861-866.

Chern, Gia Wei, Paula Mellado, and O. Tchernyshyov, 2011, "Twostage ordering of spins in dipolar spin ice on the kagome lattice," Phys. Rev. Lett. 106, 207202.

Chern, Gia-Wei, Muir J. Morrison, and Cristiano Nisoli, 2013, "Degeneracy and Criticality from Emergent Frustration in Artificial Spin Ice," Phys. Rev. Lett. 111, 177201.

Chern, Gia Wei, Charles Reichhardt, and Cristiano Nisoli, 2014, "Realizing three-dimensional artificial spin ice by stacking planar nano-arrays," Appl. Phys. Lett. 104, 013101.

Chern, Gia-Wei, C. Reichhardt, and C. J. Olson Reichhardt, 2013, "Frustrated colloidal ordering and fully packed loops in arrays of optical traps," Phys. Rev. E 87, 062305.

Chioar, I. A., Benjamin Canals, D. Lacour, M. Hehn, B. Santos Burgos, T. O. Menteş, A. Locatelli, F. Montaigne, and Nicolas Rougemaille, 2014, "Kinetic pathways to the magnetic charge crystal in artificial dipolar spin ice," Phys. Rev. B 90, 220407.

Chioar, I. A., N. Rougemaille, and B. Canals, 2016, "Ground-state candidate for the classical dipolar kagome Ising antiferromagnet," Phys. Rev. B 93, 214410.

Chioar, I. A., Nicolas Rougemaille, Alexander Grimm, Olivier Fruchart, Edouard Wagner, M. Hehn, D. Lacour, F. Montaigne, and Benjamin Canals, 2014, "Nonuniversality of artificial frustrated spin systems," Phys. Rev. B 90, 064411.

Coppersmith, S. N., Daniel S. Fisher, B. I. Halperin, P. A. Lee, and W.F. Brinkman, 1982, "Dislocations and the commensurateincommensurate transition in two dimensions," Phys. Rev. B 25, 349-363.

Cugliandolo, Leticia F., 2017, "Artificial Spin-Ice and Vertex Models," J. Stat. Phys. 167, 499-514.

Curtis, Jennifer E., Brian A. Koss, and David G. Grier, 2002, "Dynamic holographic optical tweezers," Opt. Commun. 207, 169. de las Heras, Daniel, Johannes Loehr, Michael Loenne, and Thomas M. Fischer, 2016, "Topologically protected colloidal transport above a square magnetic lattice," New J. Phys. 18, 105009.

Demirörs, Ahmet F., Pramod P. Pillai, Bartlomiej Kowalczyk, and Bartosz A. Grzybowski, 2013, "Colloidal assembly directed by virtual magnetic moulds," Nature (London) 503, 99. 
Deutschländer, Sven, Tobias Horn, Hartmut Löwen, Georg Maret, and Peter Keim, 2013, "Two-dimensional melting under quenched disorder," Phys. Rev. Lett. 111, 098301.

Drisko, Jasper, Stephen Daunheimer, and John Cumings, 2015, " $\mathrm{FePd}_{3}$ as a material for studying thermally active artificial spin ice systems," Phys. Rev. B 91, 224406.

Drisko, Jasper, Thomas Marsh, and John Cumings, 2017, "Topological frustration of artificial spin ice," Nat. Commun. 8, 14009.

Dublenych, Yu. I., 2017, "Ground states of a system of classical spins on an anisotropic triangular lattice and the spin-liquid problem in niga $_{2} \mathrm{~s}_{4}$ and fega $\mathrm{s}_{4}$ compounds," Phys. Rev. B 96, 140401.

Duplantier, Bertrand, 1998, "Random walks and quantum gravity in two dimensions," Phys. Rev. Lett. 81, 5489-5492.

Duzgun, Ayhan, and Cristiano Nisoli, 2019, "Artificial Spin Ice of Liquid Crystal Skyrmions," arXiv:1908.03246v1.

Elser, Veit, and Chen Zeng, 1993, "Kagome spin-1/2 antiferromagnets in the hyperbolic plane," Phys. Rev. B 48, 13647-13653.

Fernández-Pacheco, A., R. Streubel, O. Fruchart, R. Hertel, P. Fischer, and R. P. Cowburn, 2017, "Three-dimensional nanomagnetism," Nat. Commun. 8, 15756.

Fradkin, Eduardo, B. A. Huberman, and Stephen H. Shenker, 1978, "Gauge symmetries in random magnetic systems," Phys. Rev. B 18, 4789-4814.

Gardner, Jason S., Michel J. P. Gingras, and John E. Greedan, 2010, "Magnetic pyrochlore oxides," Rev. Mod. Phys. 82, 53-107.

Ge, Jun-Yi, Vladimir N. Gladilin, Jacques Tempere, Jozef T. Devreese, and Victor V. Moshchalkov, 2018, "Tunable and switchable magnetic dipole patterns in nanostructured superconductors," Nat. Commun. 9, 2576.

Giauque, W. F., and Muriel F. Ashley, 1933, "Molecular Rotation in Ice at $10^{\circ} \mathrm{K}$. Free Energy of Formation and Entropy of Water," Phys. Rev. 43, 81-82.

Giblin, S. R., S. T. Bramwell, P. C. W. Holdsworth, D. Prabhakaran, and I. Terry, 2011, "Creation and measurement of long-lived magnetic monopole currents in spin ice," Nat. Phys. 7, 252-258.

Gilbert, Ian, Gia-Wei Chern, Bryce Fore, Yuyang Lao, Sheng Zhang, Cristiano Nisoli, and Peter Schiffer, 2015, "Direct visualization of memory effects in artificial spin ice," Phys. Rev. B 92, 104417.

Gilbert, Ian, Gia-Wei Chern, Sheng Zhang, Liam O'Brien, Bryce Fore, Cristiano Nisoli, and Peter Schiffer, 2014, "Emergent ice rule and magnetic charge screening from vertex frustration in artificial spin ice," Nat. Phys. 10, 670-675.

Gilbert, Ian, Yuyang Lao, Isaac Carrasquillo, Liam O’Brien, Justin D. Watts, Michael Manno, Chris Leighton, Andreas Scholl, Cristiano Nisoli, and Peter Schiffer, 2016, "Emergent reduced dimensionality by vertex frustration in artificial spin ice," Nat. Phys. 12, 162-165.

Gilbert, Ian, Cristiano Nisoli, and Peter Schiffer, 2016, "Frustration by design," Phys. Today 69, No. 7, 54-59.

Glaetzle, A. W., M. Dalmonte, R. Nath, I. Rousochatzakis, R. Moessner, and P. Zoller, 2014, "Quantum spin-ice and dimer models with rydberg atoms," Phys. Rev. X 4, 041037.

Glaetzle, Alexander W., Marcello Dalmonte, Rejish Nath, Christian Gross, Immanuel Bloch, and Peter Zoller, 2015, "Designing Frustrated Quantum Magnets with Laser-Dressed Rydberg Atoms," Phys. Rev. Lett. 114, 173002.

Gliga, Sebastian, Attila Kákay, Riccardo Hertel, and Olle G. Heinonen, 2013, "Spectral Analysis of Topological Defects in an Artificial Spin-Ice Lattice," Phys. Rev. Lett. 110, 117205.

Gliga, Sebastian, et al., 2017, "Emergent dynamic chirality in a thermally driven artificial spin ratchet," Nat. Mater. 16, 1106-1111.

Glotzer, Sharon C., and Michael J. Solomon, 2007, "Anisotropy of building blocks and their assembly into complex structures," Nat. Mater. 6, 557-562.
Graser, S., P. J. Hirschfeld, T. Kopp, R. Gutser, B. M. Andersen, and J. Mannhart, 2010, "How grain boundaries limit supercurrents in high-temperature superconductors," Nat. Phys. 6, 609-614.

Grason, Gregory M., 2016, "Perspective: Geometrically frustrated assemblies,” J. Chem. Phys. 145, 110901.

Grier, David G., and Yael Roichman, 2006, "Holographic optical trapping," Appl. Opt. 45, 880.

Guerra, R. E., C. P. Kelleher, A. D. Hollingsworth, and P. M. Chaikin, 2018, "Freezing on a sphere," Nature (London) 554, 346-350.

Gunnarsson, K., P. E. Roy, S. Felton, J. Pihl, P. Svedlindh, S. Berner, H. Lidbaum, and S. Oscarsson, 2005, "Programmable motion and separation of single magnetic particles on patterned magnetic surfaces," Adv. Mater. 17, 1730.

Hall, D. M., I. R. Bruss, J.R. Barone, and G. M. Grason, 2016, "Morphology selection via geometric frustration in chiral filament bundles," Nat. Mater. 15, 727.

Han, Yilong, Yair Shokef, Ahmed M. Alsayed, Peter Yunker, Tom C. Lubensky, and Arjun G. Yodh, 2008, "Geometric frustration in buckled colloidal monolayers," Nature (London) 456, 898-903.

Harada, K., O. Kamimura, H. Kasai, T. Matsuda, A. Tonomura, and V. V. Moshchalkov, 1996, "Direct Observation of Vortex Dynamics in Superconducting Films with Regular Arrays of Defects," Science 274, 1167-1170.

Harris, M. J., S. T. Bramwell, D. F. McMorrow, T. Zeiske, and K. W. Godfrey, 1997, "Geometrical Frustration in the Ferromagnetic Pyrochlore $\mathrm{Ho}_{2} \mathrm{Ti}_{2} \mathrm{O}_{7}$," Phys. Rev. Lett. 79, 2554-2557.

Henley, Christopher L., 2010, "The Coulomb Phase in Frustrated Systems," Annu. Rev. Condens. Matter Phys. 1, 179-210.

Henley, Christopher L., 2011, "Classical height models with topological order,” J. Phys. Condens. Matter 23, 164212.

Heyderman, L. J., and R. L. Stamps, 2013, "Artificial ferroic systems: novel functionality from structure, interactions and dynamics," J. Phys. Condens. Matter 25, 363201.

Hügli, R. V., G. Duff, B. O’Conchuir, E. Mengotti, A. Fraile, F. Rodríguez, J. Nolting, L. Heyderman, and H. B. Braun, 2012, "Artificial kagome spin ice: dimensional reduction, avalanche control and emergent magnetic monopoles," Phil. Trans. R. Soc. A 370, 5767-5782.

Hynninen, A., J. Thijssen, E. C. Vermolen, M. Dijkstra, and A. van Blaaderen, 2007, "Self-assembly route for photonic crystals with a bandgap in the visible region," Nat. Mater. 6, 202-205.

Irvine, William T. M., and Vincenzo Vitelli, 2012, "Geometric background charge: dislocations on capillary bridges," Soft Matter 8, 10123-10129.

Jaubert, L. D. C., M. Haque, and R. Moessner, 2011, "Analysis of a fully packed loop model arising in a magnetic coulomb phase," Phys. Rev. Lett. 107, 177202.

Kamien, Randall D., and Jonathan V. Selinger, 2001, "Order and frustration in chiral liquid crystals," J. Phys. Condens. Matter 13, R1-R22.

Ke, X., J. Li, C. Nisoli, Paul E. Lammert, W. McConville, R. F. Wang, V. H. Crespi, and P. Schiffer, 2008, "Energy minimization and ac demagnetization in a nanomagnet array," Phys. Rev. Lett. 101, 037205.

Kosterlitz, J. M., and D. J. Thouless, 1973, “Ordering, metastability and phase transitions in two-dimensional systems," J. Phys. C 6, 1181-1203.

Kusner, R. E., J. A. Mann, J. Kerins, and A. J. Dahm, 1994, “Twostage melting of a two-dimensional collodial lattice with dipole interactions," Phys. Rev. Lett. 73, 3113-3116.

Ladak, S., D. E. Read, W. R. Branford, and L. F. Cohen, 2011, "Direct observation and control of magnetic monopole defects in an artificial spin-ice material,” New J. Phys. 13, 063032. 
Ladak, S., D.E. Read, G. K. Perkins, L.F. Cohen, and W. R. Branford, 2010, "Direct observation of magnetic monopole defects in an artificial spin-ice system," Nat. Phys. 6, 359-363.

Ladak, Sam, Dan Read, Tolek Tyliszczak, Will R. Branford, and Lesley F. Cohen, 2011, "Monopole defects and magnetic coulomb blockade," New J. Phys. 13, 023023.

Lammert, Paul E., Vincent H. Crespi, and Cristiano Nisoli, 2012, "Gibbsianizing nonequilibrium dynamics of artificial spin ice and other spin systems," New J. Phys. 14, 045009.

Lammert, Paul E., Xianglin Ke, Jie Li, Cristiano Nisoli, David M. Garand, Vincent H. Crespi, and Peter Schiffer, 2010, "Direct entropy determination and application to artificial spin ice," Nat. Phys. 6, 786-789.

Lao, Yuyang, et al., 2018, "Classical topological order in the kinetics of artificial spin ice," Nat. Phys. 14, 723-727.

Latimer, M. L., G. R. Berdiyorov, Z. L. Xiao, F. M. Peeters, and W. K. Kwok, 2013, "Realization of artificial ice systems for magnetic vortices in a superconducting moge thin film with patterned nanostructures," Phys. Rev. Lett. 111, 067001.

Lavergne, François A., Hugo Wendehenne, Tobias Bäuerle, and Clemens Bechinger, 2019, "Group formation and cohesion of active particles with visual perception dependent motility," Science 364, 70-74.

Le, B. L., et al., 2017, "Understanding magnetotransport signatures in networks of connected permalloy nanowires," Phys. Rev. B 95, 060405.

Le Cunuder, Anne, Irénée Frérot, Antonio Ortiz-Ambriz, and Pietro Tierno, 2019, "Competing orders in colloidal kagome ice: Importance of the in-trap motion of the particles," Phys. Rev. B 99, 140405.

Lee, Dong Yun, and Pietro Tierno, 2018, "Energetics and the ground state quest in an artificial triangular colloidal ice," Phys. Rev. Mater. 2, 112601 .

Lendinez, S., and M. B. Jungfleisch, 2020, "Magnetization dynamics in artificial spin ice," J. Phys. Condens. Matter 32, 013001.

Leoni, Fabio, and Yair Shokef, 2017, "Attraction controls the inversion of order by disorder in buckled colloidal monolayers," Phys. Rev. Lett. 118, 218002.

Levis, Demian, Leticia F. Cugliandolo, Laura Foini, and Marco Tarzia, 2013, "Thermal phase transitions in artificial spin ice," Phys. Rev. Lett. 110, 207206.

Li, J., S. Zhang, J. Bartell, C. Nisoli, X. Ke, Paul E. Lammert, Vincent H. Crespi, and P. Schiffer, 2010, "Comparing frustrated and unfrustrated clusters of single-domain ferromagnetic islands," Phys. Rev. B 82, 134407.

Libál, A., C. Nisoli, C. Reichhardt, and C. J. Olson Reichhardt, 2017, "Dynamic Control of Topological Defects in Artificial Colloidal Ice," Sci. Rep. 7, 651.

Libál, A., C. Nisoli, C. J. O. Reichhardt, and C. Reichhardt, 2018, "Inner phases of colloidal hexagonal spin ice," Phys. Rev. Lett. 120, 027204.

Libál, A., C. J. Olson Reichhardt, and C. Reichhardt, 2009, "Creating artificial ice states using vortices in nanostructured superconductors," Phys. Rev. Lett. 102, 237004.

Libál, A., C. J. Olson Reichhardt, and C. Reichhardt, 2015, "Doped colloidal artificial spin ice," New J. Phys. 17, 103010

Libál, A., C. Reichhardt, and C. J. Olson Reichhardt, 2006, "Realizing colloidal artificial ice on arrays of optical traps," Phys. Rev. Lett. 97, 228302.

Libál, A., C. Reichhardt, and C. J. Olson Reichhardt, 2012, "Hysteresis and return-point memory in colloidal artificial spin ice systems," Phys. Rev. E 86, 021406.
Libál, András, Dong Yun Lee, Antonio Ortiz-Ambriz, Charles Reichhardt, Cynthia J. O. Reichhardt, Pietro Tierno, and Cristiano Nisoli, 2018, "Ice rule fragility via topological charge transfer in artificial colloidal ice," Nat. Commun. 9, 4146.

Lieb, Elliott H., 1967a, "Exact solution of the $f$ model of an antiferroelectric," Phys. Rev. Lett. 18, 1046-1048.

Lieb, Elliott H., 1967b, "Residual Entropy of Square Ice," Phys. Rev. 162, 162-172.

Loehr, Johannes, Michael Loenne, Adrian Ernst, Daniel de las Heras, and Thomas M. Fischer, 2016, "Topological protection of multiparticle dissipative transport," Nat. Commun. 7, 11745.

Loehr, Johannes, Antonio Ortiz-Ambriz, and Pietro Tierno, 2016, "Defect dynamics in artificial colloidal ice: Real-time observation, manipulation, and logic gate,” Phys. Rev. Lett. 117, 168001.

Lopez-Leon, T., V. Koning, K. B. S. Devaiah, V. Vitelli, and A. Fernandez-Nieves, 2011, "Frustrated nematic order in spherical geometries," Nat. Phys. 7, 391-394.

Loreto, R. P., F. S. Nascimento, R. S. Gonçalves, J. Borme, J. C. Cezar, C. Nisoli, A. R. Pereira, and C.I. L. de Araujo, 2019, "Experimental and theoretical evidences for the ice regime in planar artificial spin ices," J. Phys. Condens. Matter 31, 025301.

Louis, D., D. Lacour, M. Hehn, V. Lomakin, Thomas Hauet, and F. Montaigne, 2018, "A tunable magnetic metamaterial based on the dipolar four-state Potts model," Nat. Mater. 17, 1076-1080.

Ma, Fusheng, C. Reichhardt, Weiliang Gan, C. J. Olson Reichhardt, and Wen Siang Lew, 2016, "Emergent geometric frustration of artificial magnetic skyrmion crystals," Phys. Rev. B 94, 144405.

Mangold, Konrad, Paul Leiderer, and Clemens Bechinger, 2003, "Phase transitions of colloidal monolayers in periodic pinning arrays," Phys. Rev. Lett. 90, 158302.

Martín, José I., M. Vélez, A. Hoffmann, Ivan K. Schuller, and J. L. Vicent, 1999, "Artificially induced reconfiguration of the vortex lattice by arrays of magnetic dots," Phys. Rev. Lett. 83, 1022-1025.

Martínez, I. A., É. Roldán, Luis Dinis, Dmitri Petrov, Juan M. R. Parrondo, and R. A. Rica, 2016, "Brownian Carnot engine," Nat. Phys. 12, 67-70.

Martínez, Ignacio A., Édgar Roldán, Luis Dinis, and Raúl A. Rica, 2017, "Colloidal heat engines: a review," Soft Matter 13, 22-36.

Massana-Cid, Helena, et al., 2019, "Edge transport at the boundary between topologically equivalent lattices," Soft Matter 15, 1539-1550.

Mazurenko, Anton, Christie S. Chiu, Geoffrey Ji, Maxwell F. Parsons, Márton Kanász-Nagy, Richard Schmidt, Fabian Grusdt, Eugene Demler, Daniel Greif, and Markus Greiner, 2017, "A cold-atom Fermi Hubbard antiferromagnet," Nature (London) 545, 462-466.

McDermott, D., J. Amelang, C. J. Olson Reichhardt, and C. Reichhardt, 2013, "Dynamic regimes for driven colloidal particles on a periodic substrate at commensurate and incommensurate fillings," Phys. Rev. E 88, 062301.

Mellado, P., O. Petrova, Y. Shen, and O. Tchernyshyov, 2010, "Dynamics of magnetic monopoles in artificial spin ice," Phys. Rev. Lett. 105, 187206.

Mellado, Paula, Andres Concha, and L. Mahadevan, 2012, "Macroscopic magnetic frustration," Phys. Rev. Lett. 109, 257203.

Mengotti, Elena, Laura J. Heyderman, Arantxa Fraile Rodríguez, Frithjof Nolting, Remo V. Hügli, and Hans-Benjamin Braun, 2011, "Real-space observation of emergent magnetic monopoles and associated Dirac strings in artificial kagome spin ice," Nat. Phys. 7, 68-74.

Mikhael, Jules, Johannes Roth, Laurent Helden, and Clemens Bechinger, 2008, "Archimedean-like tiling on decagonal quasicrystalline surfaces," Nature (London) 454, 501-504. 
Mistonov, A. A., N. A. Grigoryeva, A. V. Chumakova, H. Eckerlebe, N. A. Sapoletova, K. S. Napolskii, A. A. Eliseev, D. Menzel, and S. V. Grigoriev, 2013, "Three-dimensional artificial spin ice in nanostructured co on an inverse opal-like lattice," Phys. Rev. B 87, 220408.

Mól, L. A., R. L. Silva, R. C. Silva, A. R. Pereira, W. A. Moura-Melo, and B. V. Costa, 2009, "Magnetic monopole and string excitations in two-dimensional spin ice," J. Appl. Phys. 106, 063913.

Mól, L. A. S., A. R. Pereira, and W. A. Moura-Melo, 2012, "Extending spin ice concepts to another geometry: The artificial triangular spin ice," Phys. Rev. B 85, 184410.

Möller, G., and R. Moessner, 2006, “Artificial Square Ice and Related Dipolar Nanoarrays,” Phys. Rev. Lett. 96, 237202.

Möller, G., and R. Moessner, 2009, "Magnetic multipole analysis of kagome and artificial spin-ice dipolar arrays," Phys. Rev. B 80, 140409 .

Morfill, Gregor E., and Alexei V. Ivlev, 2009, "Complex plasmas: An interdisciplinary research field,” Rev. Mod. Phys. 81, 1353-1404.

Morgan, Jason P., Aaron Stein, Sean Langridge, and Christopher H. Marrows, 2011, "Thermal ground-state ordering and elementary excitations in artificial magnetic square ice," Nat. Phys. 7, 75-79.

Morrison, Muir J., Tammie R. Nelson, and Cristiano Nisoli, 2013, "Unhappy vertices in artificial spin ice: New degeneracies from vertex frustration," New J. Phys. 15, 045009.

Mydosh, John A., 2014, Spin glasses: an experimental introduction (CRC Press, Boca Raton).

Nakatsuji, S., Y. Nambu, H. Tonomura, O. Sakai, S. Jonas, C. Broholm, H. Tsunetsugu, Y. Qiu, and Y. Maeno, 2005, "Spin disorder on a triangular lattice," Science 309, 1697.

Nash, Lisa M., Dustin Kleckner, Alismari Read, Vincenzo Vitelli, Ari M. Turner, and William T. M. Irvine, 2015, "Topological mechanics of gyroscopic metamaterials," Proc. Natl. Acad. Sci. U.S.A. 112, 14495-14500.

Nelson, D. R., 2002, Defects and Geometry in Condensed Matter Physics Vol. 112 (Cambridge University Press, Cambridge, England).

Nishiguchi, Daiki, Igor S. Aranson, Alexey Snezhko, and Andrey Sokolov, 2018, "Engineering bacterial vortex lattice via direct laser lithography," Nat. Commun. 9, 4486.

Nisoli, Cristiano, 2012, "On thermalization of magnetic nano-arrays at fabrication," New J. Phys. 14, 035017.

Nisoli, Cristiano, 2014, "Dumping topological charges on neighbors: Ice manifolds for colloids and vortices," New J. Phys. 16, 113049.

Nisoli, Cristiano, 2016, "Nano-Ising," New J. Phys. 18, 021007.

Nisoli, Cristiano, 2018a, "Frustration (s) and the ice rule: From natural materials to the deliberate design of exotic behaviors," in Frustrated Materials and Ferroic Glasses (Springer, New York), pp. 57-99.

Nisoli, Cristiano, 2018b, "Unexpected phenomenology in particlebased ice absent in magnetic spin ice," Phys. Rev. Lett. 120, 167205.

Nisoli, Cristiano, Vassilios Kapaklis, and Peter Schiffer, 2017, "Deliberate exotic magnetism via frustration and topology," Nat. Phys. 13, 200-203.

Nisoli, Cristiano, Jie Li, Xianglin Ke, D. Garand, Peter Schiffer, and Vincent H. Crespi, 2010, "Effective temperature in an interacting vertex system: Theory and experiment on artificial spin ice," Phys. Rev. Lett. 105, 047205.

Nisoli, Cristiano, Roderich Moessner, and Peter Schiffer, 2013, "Colloquium: Artificial spin ice: Designing and imaging magnetic frustration," Rev. Mod. Phys. 85, 1473-1490.

Nisoli, Cristiano, Ruifang Wang, Jie Li, William F. McConville, Paul E. Lammert, Peter Schiffer, and Vincent H. Crespi, 2007, "Ground
State Lost but Degeneracy Found: The Effective Thermodynamics of Artificial Spin Ice,” Phys. Rev. Lett. 98, 217203.

Nixon, Micha, Eitan Ronen, Asher A. Friesem, and Nir Davidson, 2013, "Observing geometric frustration with thousands of coupled lasers," Phys. Rev. Lett. 110, 184102.

O'Holleran, Kevin, Mark R. Dennis, Florian Flossmann, and Miles J. Padgett, 2008, "Fractality of light's darkness," Phys. Rev. Lett. 100, 053902 .

Olson Reichhardt, C. J., A. Libál, and C. Reichhardt, 2012, "Multi-step ordering in kagome and square artificial spin ice," New J. Phys. 14, 025006.

Ortiz, Daniel, Kevin L. Kohlstedt, Trung Dac Nguyen, and Sharon C. Glotzer, 2014, "Self-assembly of reconfigurable colloidal molecules," Soft Matter 10, 3541.

Ortiz-Ambriz, Antonio, and Pietro Tierno, 2016, "Engineering of frustration in colloidal artificial ices realized on microfeatured grooved lattices," Nat. Commun. 7, 10575.

Östman, Erik, Henry Stopfel, Ioan-Augustin Chioar, Unnar B. Arnalds, Aaron Stein, Vassilios Kapaklis, and Björgvin Hjörvarsson, 2018, "Interaction modifiers in artificial spin ices," Nat. Phys. 14, 375.

Pauling, Linus, 1935, "The structure and entropy of ice and of other crystals with some randomness of atomic arrangement," J. Am. Chem. Soc. 57, 2680-2684.

Perrin, Yann, Benjamin Canals, and Nicolas Rougemaille, 2016, "Extensive degeneracy, Coulomb phase and magnetic monopoles in an artificial realization of the square ice model," Nature (London) 540, 410-413.

Petit, Sylvain, Elsa Lhotel, Benjamin Canals, M. Ciomaga Hatnean, Jacques Ollivier, Hannu Mutka, Eric Ressouche, A. R. Wildes, M. R. Lees, and G. Balakrishnan, 2016, "Observation of magnetic fragmentation in spin ice," Nat. Phys. 12, 746.

Phatak, C., A. K. Petford-Long, O. Heinonen, M. Tanase, and M. De Graef, 2011, "Nanoscale structure of the magnetic induction at monopole defects in artificial spin-ice lattices," Phys. Rev. B 83, 174431.

Pierce, M. S., et al., 2005, "Disorder-Induced Microscopic Magnetic Memory," Phys. Rev. Lett. 94, 017202.

Pollard, S. D., V. Volkov, and Y. Zhu, 2012, "Propagation of magnetic charge monopoles and Dirac flux strings in an artificial spin-ice lattice," Phys. Rev. B 85, 180402.

Poon, Wilson, 2004, "PHYSICS: Colloids as Big Atoms," Science 304, 830-831.

Porro, J. M., A. Bedoya-Pinto, A. Berger, and P. Vavassori, 2013, "Exploring thermally induced states in square artificial spin-ice arrays," New J. Phys. 15, 055012.

Pyka, K., et al., 2013, "Topological defect formation and spontaneous symmetry breaking in ion Coulomb crystals," Nat. Commun. 4, 2291.

Qi, Yi, T. Brintlinger, and John Cumings, 2008, "Direct observation of the ice rule in an artificial kagome spin ice," Phys. Rev. B 77, 094418.

Ramirez, A. P., 1994, "Strongly Geometrically Frustrated Magnets," Annu. Rev. Mater. Sci. 24, 453-480.

Ramirez, A. P., A. Hayashi, R. J. Cava, R. Siddharthan, and B. S. Shastry, 1999, "Zero-point entropy in spin ice'," Nature (London) 399, 333-335.

Reichhardt, C., and C. J. Olson, 2002, "Novel colloidal crystalline states on two-dimensional periodic substrates," Phys. Rev. Lett. 88, 248301.

Reichhardt, C., C. J. Olson, N. Grønbech-Jensen, and Franco Nori, 2001, "Moving Wigner Glasses and Smectics: Dynamics of Disordered Wigner Crystals," Phys. Rev. Lett. 86, 4354-4357. 
Ribeiro, I. R. B., F. S. Nascimento, S. O. Ferreira, W. A. MouraMelo, C. A. R. Costa, J. Borme, P. P. Freitas, G. M. Wysin, C. I. L. De Araujo, and A. R. Pereira, 2017, "Realization of Rectangular Artificial Spin Ice and Direct Observation of High Energy Topology," Sci. Rep. 7, 13982.

Richard, Patrick, Mario Nicodemi, Renaud Delannay, Philippe Ribière, and Daniel Bideau, 2005, "Slow relaxation and compaction of granular systems," Nat. Mater. 4, 121-128.

Rivnay, J., L. H. Jimison, J. E. Northrup, and M. F. Toney, 2009, "Large modulation of carrier transport by grain-boundary molecular packing and ...," Nat. Mater. 8, 952-958.

Rodrigues, J. H., L. A. S. Mól, W. A. Moura-Melo, and A. R. Pereira, 2013, "Efficient demagnetization protocol for the artificial triangular spin ice," Appl. Phys. Lett. 103, 092403.

Rougemaille, N., and B. Canals, 2019, "Cooperative magnetic phenomena in artificial spin systems: spin liquids, Coulomb phase and fragmentation of magnetism - a colloquium," Eur. Phys. J. B 92, 62.

Rougemaille, N., et al., 2011, "Artificial Kagome Arrays of Nanomagnets: A Frozen Dipolar Spin Ice,” Phys. Rev. Lett. 106, 057209.

Rys, Franz, 1963, Ueber ein zweidimensionales klassisches Konfigurationsmodell, Ph.D. thesis (ETH Zürich).

Ryzhkin, I. A., 2005, "On magnetic relaxation in rare earth metal perchlorate metals," Zh. Eksp. Teor. Fiz. 128, 559-566.

Šarlah, A., T. Franosch, and E. Frey, 2005, "Melting of colloidal molecular crystals on triangular lattices," Phys. Rev. Lett. 95, 088302.

Schall, Peter, 2004, "Visualization of Dislocation Dynamics in Colloidal Crystals," Science 305, 1944-1948.

Schall, Peter, David A. Weitz, and Frans Spaepen, 2007, "Structural Rearrangements That Govern Flow in Colloidal Glasses," Science 318, 1895-1899.

Sen, Arnab, and R. Moessner, 2015, "Topological spin glass in diluted spin ice,” Phys. Rev. Lett. 114, 247207.

Sendetskyi, Oles, Valerio Scagnoli, Naëmi Leo, Luca Anghinolfi, Aurora Alberca, Jan Lüning, Urs Staub, Peter Michael Derlet, and Laura Jane Heyderman, 2019, "Continuous magnetic phase transition in artificial square ice," Phys. Rev. B 99, 214430.

Shi, Dong, Zoe Budrikis, Aaron Stein, Sophie A. Morley, Peter D. Olmsted, Gavin Burnell, and Christopher H. Marrows, 2018, "Frustration and thermalization in an artificial magnetic quasicrystal," Nat. Phys. 14, 309-314.

Shokef, Y., A. Souslov, and T. C. Lubensky, 2011, "Order by disorder in the antiferromagnetic Ising model on an elastic triangular lattice," Proc. Natl. Acad. Sci. U.S.A. 108, 11804-11809.

Shokef, Yair, Yilong Han, Anton Souslov, A. G. Yodh, and Tom C. Lubensky, 2013, "Buckled colloidal monolayers connect geometric frustration in soft and hard matter," Soft Matter 9, 6565-6570.

Silva, R. C., R. J. C. Lopes, L. A. S. Mól, W. A. Moura-Melo, G. M. Wysin, and A. R. Pereira, 2013, "Nambu monopoles interacting with lattice defects in a two-dimensional artificial square spin ice," Phys. Rev. B 87, 014414.

Skjaervø, S. H., et al., 2019, "Advances in artificial spin ice," Nat. Rev. Phys.

Sklenar, Joseph, Yuyang Lao, Alan Albrecht, Justin D. Watts, Cristiano Nisoli, Gia-Wei Chern, and Peter Schiffer, 2019, "Field-induced phase coexistence in an artificial spin ice," Nat. Phys. 15, 191-195.

Soni, Vishal, Leopoldo R. Gómez, and William T. M. Irvine, 2018, "Emergent geometry of inhomogeneous planar crystals," Phys. Rev. X 8, 011039.
Stout, J. W., and W. F. Giauque, 1936, "The Entropy of Water and the Third Law of Thermodynamics. The Heat Capacity of Ice from 15 to $273^{\circ} \mathrm{K}, "$ J. Am. Chem. Soc. 58, 1144-1150.

Tanaka, M., E. Saitoh, H. Miyajima, T. Yamaoka, and Y. Iye, 2006, "Magnetic interactions in a ferromagnetic honeycomb nanoscale network," Phys. Rev. B 73, 052411.

Thorneywork, Alice L., Joshua L. Abbott, Dirk G. A. L. Aarts, and Roel P. A. Dullens, 2017, "Two-dimensional melting of colloidal hard spheres," Phys. Rev. Lett. 118, 158001.

Tierno, Pietro, 2012, "Depinning and collective dynamics of magnetically driven colloidal monolayers," Phys. Rev. Lett. 109, 198304.

Tierno, Pietro, 2014, "Magnetic assembly and annealing of colloidal lattices and superlattices," Langmuir 30, 7670.

Tierno, Pietro, 2016, "Geometric frustration of colloidal dimers on a honeycomb magnetic lattice," Phys. Rev. Lett. 116, 038303.

Tierno, Pietro, and Thomas M. Fischer, 2014, "Excluded volume causes integer and fractional plateaus in colloidal ratchet currents," Phys. Rev. Lett. 112, 048302.

Tierno, Pietro, Thomas M. Fischer, Tom H. Johansen, and Francesc Sagués, 2008, "Colloidal assembly on magnetically vibrated stripes," Phys. Rev. Lett. 100, 148304.

Tierno, Pietro, Tom H. Johansen, and Thomas M. Fischer, 2007, "Localized and delocalized motion of colloidal particles on a magnetic bubble lattice,” Phys. Rev. Lett. 99, 038303.

Tierno, Pietro, Francesc Sagués, Tom H. Johansen, and Thomas M. Fischer, 2009, "Colloidal transport on magnetic garnet films," Phys. Chem. Chem. Phys. 11, 9615.

Trastoy, J., M. Malnou, C. Ulysse, R. Bernard, N. Bergeal, G. Faini, J. Lesueur, J. Briatico, and Javier E. Villegas, 2014, "Freezing and thawing of artificial ice by thermal switching of geometric frustration in magnetic flux lattices," Nat. Nanotechnol. 9, 710-715.

Trastoy, J., C. Ulysse, R. Bernard, M. Malnou, N. Bergeal, J. Lesueur, J. Briatico, and Javier E. Villegas, 2015, "Tunable Flux-Matching Effects in High- $T_{c}$ Superconductors with Nonuniform Pinning Arrays," Phys. Rev. Applied 4, 054003.

Tung, S., V. Schweikhard, and E. A. Cornell, 2006, "Observation of vortex pinning in bose-einstein condensates," Phys. Rev. Lett. 97, 240402.

van der Zande, Arend M., Pinshane Y. Huang, Daniel a. Chenet, Timothy C. Berkelbach, YuMeng You, Gwan-Hyoung Lee, Tony F. Heinz, David R. Reichman, David a. Muller, and James C. Hone, 2013, "Supplementary Information for Grains and grain boundaries in highly crystalline monolayer molybdenum disulphid," Nat. Mater. 12, 554-561.

Vanossi, A., N. Manini, and E. Tosatti, 2012, "Static and dynamic friction in sliding colloidal monolayers," Proc. Natl. Acad. Sci. U.S.A. 109, 16429-16433.

Vedmedenko, E. Y., 2016, "Dynamics of Bound Monopoles in Artificial Spin Ice: How to Store Energy in Dirac Strings," Phys. Rev. Lett. 116, 077202.

Wang, R. F., et al., 2006, "Artificial 'spin ice' in a geometrically frustrated lattice of nanoscale ferromagnetic islands," Nature (London) 439, 303-306.

Wang, Y.-L., et al., 2016, "Rewritable artificial magnetic charge ice," Science 352, 962-966.

Wang, Yong Lei, Xiaoyu Ma, Jing Xu, Zhi Li Xiao, Alexey Snezhko, Ralu Divan, Leonidas E. Ocola, John E. Pearson, Boldizsar Janko, and Wai Kwong Kwok, 2018, "Switchable geometric frustration in an artificial-spin-ice-superconductor heterosystem," Nat. Nanotechnol. 13, 560-565.

Wang, Yufeng, Yufeng Wang, Dana R. Breed, Vinothan N. Manoharan, Lang Feng, Andrew D. Hollingsworth, Marcus Weck, 
and David J. Pine, 2012, "Colloids with valence and specific directional bonding," Nature (London) 491, 51-55.

Wannier, G. H., 1950, "Antiferromagnetism. The Triangular Ising Net," Phys. Rev. 79, 357-364.

Wannier, G. H., 1973, "Antiferromagnetism. the triangular ising net," Phys. Rev. B 7, 5017.

Weeks, Eric R., 2017, "Introduction to the colloidal glass transition," ACS Macro Lett. 6, 27-34.

Wioland, Hugo, Francis G. Woodhouse, Jörn Dunkel, and Raymond E. Goldstein, 2016, "Ferromagnetic and antiferromagnetic order in bacterial vortex lattices," Nat. Phys. 12, 341-345.

Wu, F. Y., 1969, "Critical Behavior of Two-Dimensional HydrogenBonded Antiferroelectrics," Phys. Rev. Lett. 22, 1174-1176.

Xue, C., J.-Y. Ge, A. He, V. S. Zharinov, V. V. Moshchalkov, Y. H. Zhou, A. V. Silhanek, and J. Van de Vondel, 2017, "Mapping degenerate vortex states in a kagome lattice of elongated antidots via scanning hall probe microscopy," Phys. Rev. B 96, 024510.

Xue, Cun, Jun-Yi Ge, An He, Vyacheslav S. Zharinov, Victor V. Moshchalkov, and You-He Zhou, 2018, "Stability of degenerate vortex states and multi-quanta confinement effects in a nanostructured superconductor with Kagome lattice of elongated antidots," New J. Phys. 20, 093030.

Yellen, B. B., O. Hovorka, and G. Friedman, 2005, "Arranging matter by magnetic nanoparticle assemblers," Proc. Natl. Acad. Sci. U.S.A. 102, 8860.

Yethiraj, Anand, and Alfons van Blaaderen, 2003, "A colloidal model system with an interaction tunable from hard sphere to soft and dipolar," Nature (London) 421, 513-517.
Zahn, K., R. Lenke, and G. Maret, 1999, "Two-stage melting of paramagnetic colloidal crystals in two dimensions," Phys. Rev. Lett. 82, 2721-2724.

Zakhidov, Anvar A., Ray H. Baughman, Zafar Iqbal, Changxing Cui, Ilyas Khayrullin, Socrates O. Dantas, Jordi Marti, and Victor G. Ralchenko, 1998, "Carbon structures with three-dimensional periodicity at optical wavelengths," Science 282, 897-901.

Zeissler, K., S. K. Walton, S. Ladak, D. E. Read, T. Tyliszczak, L. F. Cohen, and W. R. Branford, 2013, "The non-random walk of chiral magnetic charge carriers in artificial spin ice," Sci. Rep. 3, 1252.

Zhang, Sheng, Ian Gilbert, Cristiano Nisoli, Gia Wei Chern, Michael J. Erickson, Liam O'Brien, Chris Leighton, Paul E. Lammert, Vincent H. Crespi, and Peter Schiffer, 2013, "Crystallites of magnetic charges in artificial spin ice," Nature (London) 500, 553-557.

Zhang, Sheng, et al., 2012, "Perpendicular magnetization and generic realization of the ising model in artificial spin ice," Phys. Rev. Lett. 109, 087201.

Zhao, Kun, and Thomas G. Mason, 2009, "Frustrated rotator crystals and glasses of brownian pentagons," Phys. Rev. Lett. 103, 208302.

Zhao, Kun, and Thomas G. Mason, 2015, "Shape-designed frustration by local polymorphism in a near-equilibrium colloidal glass," Proc. Natl. Acad. Sci. U.S.A. 112, 12063-12068.

Zhou, Di, Feng Wang, Bo Li, Xiaojie Lou, and Yilong Han, 2017, "Glassy spin dynamics in geometrically frustrated buckled colloidal crystals," Phys. Rev. X 7, 021030. 